\title{
Pionic dark matter
}

\author{
Subhaditya Bhattacharya, ${ }^{a, 1}$ Blaženka Melić ${ }^{a, b}$ and José Wudka ${ }^{a}$ \\ ${ }^{a}$ Department of Physics \& Astronomy, University of California Riverside, \\ Riverside, CA 92521-0413, U.S.A. \\ ${ }^{b}$ Rudjer Bošković Institute, Theoretical Physics Division, \\ P.O.Box 180, HR-10002 Zagreb, Croatia \\ E-mail: subhaditya.bhattacharya@ucr.edu, blazenka.melic@irb.hr, \\ jose.wudka@ucr.edu
}

ABSTRACT: We study a phenomenological model where the lightest dark matter (DM) particles are the pseudo-Goldstone excitations associated with a spontaneously broken symmetry, and transforming linearly with respect to an unbroken group $\mathcal{H}_{\mathrm{DM}}$. For definiteness we take $\mathcal{H}_{\mathrm{DM}}=\mathrm{SU}(N)$ and assume the Goldstone particles are bosons; in parallel with QCD, we refer to these particles as dark-matter pions. This scenario is in contrast to the common assumption that DM fields transform linearly under the full symmetry of the model. We illustrate the formalism by treating in detail the case of $\mathcal{H}_{\mathrm{DM}}=\mathrm{SU}(2)$, in particular we calculate all the interactions relevant for the Boltzmann equations, which we solve numerically; we also derive approximate analytic solutions and show their consistency with the numerical results. We then compare the results with the constraints derived from the cold DM and direct detection experiments and derive the corresponding restrictions on the model parameters. We also briefly comment on constraints from indirect detection of DM.

KEYwords: Beyond Standard Model, Cosmology of Theories beyond the SM

ARXIV EPRINT: 1307.2647

\footnotetext{
${ }^{1}$ Corresponding author.
} 


\section{Contents}

1 Introduction 1

2 Nonlinear realization of $\mathcal{G}_{\mathrm{DM}} \quad 2$

2.1 Conserved currents 6

$\begin{array}{ll}2.2 & \text { Parameters of the model } \\ \end{array}$

3 DMP interactions $\quad 8$

3.1 DMP $\rightarrow$ SM interactions 8

$\begin{array}{lll}3.2 & \text { Direct-detection reaction } & 10\end{array}$

3.3 Pure DMP scattering 11

3.4 Decays of SM particles to DMP 12

4 Thermal history of DMP 13

$\begin{array}{lll}4.1 & \text { Boltzmann equations } & 13\end{array}$

$\begin{array}{lll}4.2 & \text { Contributions from } \mathrm{SM} \rightarrow \text { DMP decays } & 16\end{array}$

5 Solving the Boltzmann equations for the $\mathrm{SU}(2)$ case 17

$\begin{array}{ll}5.1 \text { Zero charge solutions } & 20\end{array}$

5.2 Behavior for small values of $|q| \quad 21$

$6 \quad$ Experimental limits on model parameters $\quad 23$

6.1 Constraints from the cold dark matter (CDM) relic density measurements 24

6.2 Direct detection constraints 26

6.3 Combined constraints on DMP model 27

6.4 Comparison with the standard Higgs-portal results 29

$\begin{array}{lll}7 & \text { Conclusions } & 30\end{array}$

A Effects on the Boltzmann equations of the SM particle decays to DMP. 31

B Kinetics of pure DMP

\section{Introduction}

Dark matter (DM) is the most promising hypothesis proposed to explain astrophysical and cosmological observations related to the motion of stars in galaxies [1], the motion of galaxies in clusters [2-4], structure formation [5] and the inhomogeneities in the CMBR [6, 7]. Having not direct experimental information about this component of the universe the theoretical efforts to understand DM have been couched within realistic extensions of the Standard model (SM) [8-14], or have taken a purely phenomenological approach [15-23], in which case simplicity has been used as a guide and constraint. 
In this publication we will investigate a phenomenological model for DM based on general assumptions concerning the dark sector, explicitly, we will assume that the lightest particles in that sector are the pseudo-Goldstone bosons resulting from a broken symmetry [24]. Operationally this implies that the lightest particles (that we take as scalars for simplicity) transform non-linearly under a continuous symmetry group, a situation similar to the one occurring in low energy hadron physics. Accordingly, we will refer to them as dark matter pions (DMP) (we emphasize however, that these are quite distinct form the pions in the hadronic sector, in particular they do not have direct couplings to the standard model (SM) $W^{ \pm}$and photon, and in that sense our assumptions fundamentally differ from those made in [25]). This approach is in contrast with most phenomenological approaches where the dark-sector fields are assumed to transform under a discrete symmetry, or linearly under a continuous one $[15-18] .^{1}$

In the following we will study this type of DM model based on the nonlinear realization of a spontaneously broken symmetry group $\mathcal{G}_{\text {DM }}$. However, given the difficulties of hot dark matter gas in dealing with structure formation [26-28], we will also assume that the Goldstone bosons receive their masses through an explicit breaking of the original symmetry. We also require that all SM particles are singlets under the dark-sector symmetries and that the dark particles are singlets under the SM local symmetries.

A similar approach has been followed in several publications. The use of NambuGoldstone bosons as DM was studied in [29, 30], their stabilization using G parity in [31, 32] and the possibility of composite DM in [33, 34].

The interaction between these two sectors (SM and DM) is presumably effected by the exchange of some heavy mediators whose nature we do not need to specify, but only assume are much heavier than the typical scales in either sector. ${ }^{2}$ Therefore the typical interactions are of the form

$$
\mathcal{L}_{\mathrm{DM}-\mathrm{SM}} \sim \frac{1}{\Lambda^{n}} \mathcal{O}_{\mathrm{DM}} \mathcal{O}_{\mathrm{SM}}
$$

where $\mathcal{O}_{D M}, \mathcal{O}_{\mathrm{SM}}$ are operators invariant under the internal symmetries of the corresponding sector, but they need not be Lorentz invariant (though, of course, $\mathcal{L}_{\mathrm{DM}-\mathrm{SM}}$ must be). The details of these interactions will be elaborated below.

This paper is organized as follows: in the next section we describe the formalism behind our model, and construct the Lagrangian we will use in our calculations. In section 3 we calculate the SM-DM interactions that we then use in sections 4 and 5 to derive the relic abundance of this type of dark matter. These results are compared with the experimental constraints in section 6 with our brief conclusions are presented in section 7 . A few details are relegated to the two appendices.

\section{Nonlinear realization of $\mathcal{G}_{\mathrm{DM}}$}

Models where the symmetry is non-linearly realized have been extensively studied (see, e.g. $[35,36])$; here we summarize some of the results for completeness. We assume there is a subgroup $\mathcal{H}_{\mathrm{DM}} \subset \mathcal{G}_{\mathrm{DM}}$ under which the vacuum is invariant and, following [35, 36], we

\footnotetext{
${ }^{1}$ The model studied here also respects several discrete $Z_{2}$ symmetries, whose presence follows from the required behavior under $\mathcal{G}_{\mathrm{DM}}$ and $\mathcal{H}_{\mathrm{DM}}$.

${ }^{2}$ Explicit realization of such mediators are discussed in [29, 30].
} 
denote the generators of $\mathcal{H}_{\mathrm{DM}}$ by $V_{i}$ and the remaining generators of $\mathcal{G}_{\mathrm{DM}}$ by $T_{a}$. Then the fields can be chosen as $\{\boldsymbol{\pi}, \boldsymbol{\psi}\}$ with the following properties:

- Under $\mathcal{H}_{\mathrm{DM}}$ they transform linearly: $\boldsymbol{\pi} \rightarrow \mathcal{D}(h) \boldsymbol{\pi}, \boldsymbol{\psi} \rightarrow D(h) \boldsymbol{\psi}$ for $h \in \mathcal{H}_{\mathrm{DM}}$; where $\mathcal{D}$ and $D$ are some matrix representations of $\mathcal{H}_{\mathrm{DM}}$.

- Under a general $g \in \mathcal{G}_{\mathrm{DM}}$

$$
\boldsymbol{\pi} \rightarrow \boldsymbol{\xi}(\boldsymbol{\pi}, g), \quad \boldsymbol{\psi} \rightarrow D\left(e^{\mathbf{u} \cdot \mathbf{V}}\right) \boldsymbol{\psi} ; \mathbf{u}=\mathbf{u}(\boldsymbol{\pi}, g)
$$

where $D$ is the same representation as above, and $\boldsymbol{\xi}$ and $\mathbf{u}$ are defined by

$$
g e^{\boldsymbol{\pi} \cdot \mathbf{T}}=e^{\boldsymbol{\xi} \cdot \mathbf{T}} e^{\mathbf{u} \cdot \mathbf{V}} .
$$

Note that the transformation of $\boldsymbol{\pi}$ depends only on $g$ and $\boldsymbol{\pi}$, and is non-linear; while that of $\boldsymbol{\psi}$ depends on $g, \boldsymbol{\psi}$ and $\boldsymbol{\pi}$. Because of their transformation properties the $\boldsymbol{\pi}$ are massless and correspond to the Goldstone bosons generated under the spontaneous breaking $\mathcal{G}_{\mathrm{DM}} \rightarrow \mathcal{H}_{\mathrm{DM}}$, and accordingly the number of these fields equals that of the broken generators $T_{a}$. We will refer to the $\boldsymbol{\pi}$ as the "dark-matter pions" (DMP) or dark pions.

To be specific we concentrate on the familiar case [37-40] of a unitary chiral theory where $\mathcal{G}_{\mathrm{DM}}=\mathrm{SU}(N) \times \mathrm{SU}(N)$ and $\mathcal{H}_{\mathrm{DM}}=\mathrm{SU}(N)$, the diagonal subgroup. In this case the above general formalism is realized by introducing a unitary field $\Sigma$ and transforms as

$$
\Sigma \rightarrow L \Sigma R^{\dagger} \quad L, R \in \mathrm{SU}(N)
$$

where $\Sigma=\exp (i \boldsymbol{\pi} \cdot \mathbf{T} / f)$ and $f$ is a mass scale associated with the spontaneous breaking of the symmetry. The diagonal subgroup corresponds to the choice $R=L$.

As it is well known [39-41], the leading fully chirally invariant operator is

$$
\mathcal{L}^{(0)}=f^{2} \operatorname{tr}\left\{\partial_{\mu} \Sigma^{\dagger} \partial^{\mu} \Sigma\right\}
$$

Expanding (2.4) in terms of the $\boldsymbol{\pi}$ we find that this Lagrangian describes a series of massless particles ${ }^{3}$ which are difficult (though not impossible [45]) to reconcile with structure formation. We will therefore also include an explicit breaking of the $\mathcal{G}_{\mathrm{DM}}$ symmetry that generate a mass for these excitations; for the chiral model this corresponds to a term of the form

$$
\mathcal{L}_{\text {mass }}=\frac{1}{2} f^{2}\left(M^{2} \operatorname{tr}\{\Sigma\}+\text { H.c. }\right) .
$$

This term is invariant under the diagonal (unbroken) subgroup $\mathcal{H}_{\mathrm{DM}}$ and gives the same mass $M$ to all the $\boldsymbol{\pi}$; we could have used a more general mass matrix that breaks $\mathcal{H}_{\mathrm{DM}}$ explicitly. If this breaking is small compared to $M$ this generalization would not change qualitatively the results below. If the breaking is large the heavy $\pi$ would become unstable against decaying into the lighter ones and the picture presented below breaks down. Here we will only consider the fully degenerate case for simplicity.

\footnotetext{
${ }^{3}$ We will not be concerned here with coherent excitations that might be stabilized by higher-derivative operators that describe dark baryons [42-44].
} 
In order to construct the DM-SM interactions of the form (1.1) we need the list of the lowest-dimensional SM gauge-invariant (tough not necessarily Lorentz invariant) operators. These are easily listed; for dimension $\leq 2$ we have

$$
\operatorname{dim} 2:|\phi|^{2}, B_{\mu \nu},
$$

where $\phi$ denotes the SM scalar doublet and $B$ the hypercharge gauge field containing physical $Z$ and $\gamma$ bosons. The dimension 3 operators (that we will not use here) are $\phi^{\dagger} D_{\mu} \phi$ and $\bar{\psi} \gamma_{\mu} \psi^{\prime}$, where $\psi$ and $\psi^{\prime}$ are any two fermion fields carrying the same gauge group representation (e.g. $e_{R}$ and $\tau_{R}$ ); higher dimensional operators are similarly constructed.

Then, the simplest DM-SM coupling is clearly

$$
\mathcal{L}_{\Sigma-\phi}=\frac{1}{2} \lambda_{h}\left(|\phi|^{2}-v^{2}\right) \operatorname{tr}\left\{\partial_{\mu} \Sigma^{\dagger} \partial^{\mu} \Sigma\right\}
$$

where $v=\langle\phi\rangle \sim 174 \mathrm{GeV}$.

The coupling $\Sigma$ to $B_{\mu \nu}$ is less straightforward since there are no $\mathcal{G}_{\mathrm{DM}}$-invariant operators that can be constructed out of $\Sigma$ and its derivatives and which transforms as the $(0,1)+(1,0)$ representation of the Lorentz group. ${ }^{4}$ Noting however, that $(2.5)$ is invariant only under the diagonal subgroup $\mathcal{H}_{\mathrm{DM}}$, we will only require the $\Sigma-B$ coupling to have the same property, and in this case,

$$
\mathcal{L}_{\Sigma-B}=B^{\mu \nu}\left(\lambda_{V} \operatorname{tr}\left\{\Sigma^{\dagger} \partial_{\mu} \Sigma \partial_{\nu} \Sigma^{\dagger}\right\}+\text { H.c. }\right) .
$$

For our choices of $\mathcal{G}_{\mathrm{DM}}$ and $\mathcal{H}_{\mathrm{DM}}$ the Lagrangian for our model is obtained from (2.4), (2.5), (2.7), (2.8); explicitly,

$$
\begin{aligned}
\mathcal{L}= & \frac{1}{2}\left[f^{2}+\lambda_{h}\left(|\phi|^{2}-v^{2}\right)\right] \operatorname{tr}\left\{\partial_{\mu} \Sigma^{\dagger} \partial^{\mu} \Sigma\right\} \\
& +\frac{1}{2} f^{2}\left(M^{2} \operatorname{tr}\{\Sigma\}+\text { H.c. }\right)+B^{\mu \nu}\left(\lambda_{V} \operatorname{tr}\left\{\Sigma^{\dagger} \partial_{\mu} \Sigma \partial_{\nu} \Sigma^{\dagger}\right\}+\text { H.c. }\right)
\end{aligned}
$$

where, as before,

$$
\Sigma=\exp \left(\frac{i}{f} \pi_{a} T_{a}\right)
$$

In parallel with the usual strong-interaction pions, we will call $f$ the DMP decay constant.

The $T_{a}$ are the broken Hermitian generators normalized by

$$
\operatorname{tr}\left\{T_{a} T_{b}\right\}=\delta_{a b},
$$

and obeying

$$
\left[T_{a}, T_{b}\right]=i f_{a b c} T_{c}
$$

(with $a, b, \ldots=1,2, \ldots, N^{2}-1$ ). In the Cartan basis with root generators $T_{ \pm \boldsymbol{\alpha}}$ and Cartan generators $T_{i}$ we have $[46,47]$

$$
\left[T_{i}, T_{j}\right]=0, \quad\left[T_{i}, T_{\boldsymbol{\alpha}}\right]=\alpha_{i} T_{\boldsymbol{\alpha}}, \quad\left[T_{\boldsymbol{\alpha}}, T_{\boldsymbol{\beta}}\right]=N_{\boldsymbol{\alpha}, \boldsymbol{\beta}} T_{\boldsymbol{\alpha}+\boldsymbol{\beta}}
$$

where $N_{\boldsymbol{\alpha}, \boldsymbol{\beta}}=0$ if $\boldsymbol{\alpha}+\boldsymbol{\beta}$ is not a root.

\footnotetext{
${ }^{4}$ Those terms become available for models with two chiral fields $\Sigma_{1,2}$ that transform in the same way.
} 
We could also add another $\phi-\pi$ coupling by replacing

$$
M^{2} \rightarrow M^{2}(\phi)=M^{2}+\lambda_{h}^{\prime}\left(|\phi|^{2}-v^{2}\right) .
$$

To lowest order this coupling is of the form $|\phi|^{2} \pi^{2}$ and its effects have been studied extensively [48, 49]. Given our interest in studying the effects of the new interactions listed in (2.9) we will neglect $\lambda_{h}^{\prime}$ in the following. We will, however, present in section 6.4 a brief discussion of the case where $\lambda_{h}^{\prime} \neq 0$ and $\lambda_{h}=\lambda_{V}=0$.

Writing $\Sigma=\exp (i \sigma)$ and using

$$
\delta \Sigma=i \int_{0}^{1} d u e^{i(1-u) \sigma} \delta \sigma e^{i u \sigma}, \quad \sigma=\boldsymbol{\pi} . \mathbf{T} / f
$$

the Lagrangian can be written (in a Hermitian basis)

$$
\begin{aligned}
\mathcal{L}= & \frac{1}{2}\left(1+\lambda_{h} \frac{|\phi|^{2}-v^{2}}{f^{2}}\right) \partial_{\mu} \pi_{a} \partial^{\mu} \pi_{b} g_{a b}+\frac{1}{2} M^{2} f^{2} \operatorname{tr}\left\{\Sigma+\Sigma^{\dagger}\right\} \\
& -\frac{1}{f^{2}} B^{\mu \nu} \partial_{\mu} \pi_{a} \partial_{\nu} \pi_{b} g_{a c} f_{c b d} \operatorname{Im}\left(\lambda_{V} \operatorname{tr}\left\{T_{d} \Sigma^{\dagger}\right\}\right) \\
= & \frac{1}{2}(\partial \boldsymbol{\pi})^{2}-\frac{1}{2} M^{2} \boldsymbol{\pi}^{2}+\frac{\lambda_{h} v}{\sqrt{2} f^{2}} h(\partial \boldsymbol{\pi})^{2}+\frac{\lambda_{h}}{4 f^{2}} h^{2}(\partial \boldsymbol{\pi})^{2}-\frac{\operatorname{Re}\left(\lambda_{V}\right)}{f^{3}} B^{\mu \nu} f_{a b c} \partial_{\mu} \pi_{a} \partial_{\nu} \pi_{b} \pi_{c}+\cdots,
\end{aligned}
$$

where

$$
g_{a b}=\int_{-1}^{1} d u(1-|u|) \operatorname{tr}\left\{e^{i u \sigma} T_{a} e^{-i u \sigma} T_{b}\right\}
$$

and $h$ is the Higgs field; in unitary $(\mathrm{SM})$ gauge $\phi^{T}=(v+h / \sqrt{2})(0,1)$.

In the Cartan basis,

$$
\begin{aligned}
\boldsymbol{\pi}^{2} & =\sum_{i} \pi_{i}^{2}+\sum_{\boldsymbol{\alpha}}\left|\pi_{\boldsymbol{\alpha}}\right|^{2}, \quad \pi_{-\boldsymbol{\alpha}}=\pi_{\boldsymbol{\alpha}}^{\dagger} \\
(\partial \boldsymbol{\pi})^{2} & =\sum_{i}\left(\partial \pi_{i}\right)^{2}+\sum_{\boldsymbol{\alpha}}\left|\partial \pi_{\boldsymbol{\alpha}}\right|^{2}=\sum_{i}\left(\partial \pi_{i}\right)^{2}+2 \sum_{\boldsymbol{\alpha}>0}\left|\partial \pi_{\boldsymbol{\alpha}}\right|^{2}, \\
B^{\mu \nu} f_{a b c} \partial_{\mu} \pi_{a} \partial_{\nu} \pi_{b} \pi_{c}=i B^{\mu \nu} & {\left[\sum_{\boldsymbol{\alpha}, \boldsymbol{\beta}} N_{\boldsymbol{\alpha}, \boldsymbol{\beta}} \pi_{\boldsymbol{\alpha}+\boldsymbol{\beta}}^{\dagger} \partial_{\mu} \pi_{\boldsymbol{\alpha}} \partial_{\nu} \pi_{\boldsymbol{\beta}}\right.} \\
& \left.+\sum_{i, \boldsymbol{\alpha}} \alpha_{i} \partial_{\nu} \pi_{\boldsymbol{\alpha}}^{\dagger}\left(2 \pi_{\boldsymbol{\alpha}} \partial_{\mu} \pi_{i}-\pi_{i} \partial_{\mu} \pi_{\boldsymbol{\alpha}}\right)\right]
\end{aligned}
$$

and for the case of $N=2$ (that we will develop later as a specific illustrative case):

$$
\begin{aligned}
\boldsymbol{\pi}^{2} & =\pi_{o}^{2}+2 \pi_{+} \pi_{-}, \\
(\partial \boldsymbol{\pi})^{2} & =\left(\partial \pi_{o}\right)^{2}+2 \partial \pi_{+} \partial \pi_{-}, \\
B^{\mu \nu} f_{a b c} \partial_{\mu} \pi_{a} \partial_{\nu} \pi_{b} \pi_{c} & =-2 i B^{\mu \nu}\left[\left(\partial_{\mu} \pi_{o}\right)\left(\pi_{-} \stackrel{\leftrightarrow}{\partial} \pi_{+}\right)+\pi_{o} \partial_{\mu} \pi_{+} \partial_{\nu} \pi_{-}\right],
\end{aligned}
$$

where $\pi_{o}$ is associated with the $\mathrm{SU}(2)$ Cartan generator, and $\pi_{ \pm}=\pi_{ \pm \boldsymbol{\alpha}}$, where $\boldsymbol{\alpha}$ is the single root in this group. 
It is important to note that despite the presence of a vertex of $Z$ (residing in the $B_{\mu \nu}$ field in (2.19)) with an odd number of $\pi$, the dark pions are stable: their decay to other DMPs is kinematically forbidden, while decay to SM particles is forbidden by the exact $\mathcal{H}_{\text {DM }}$ symmetry under which the DMP are triplets, while all SM states are singlets. This, of course, would be modified if we were to allow $\mathcal{H}_{\mathrm{DM}}$-violating terms with sufficiently large coefficients. We restrict ourselves to regions in parameter space where this does not occur (e.g. where $\mathcal{H}_{\mathrm{DM}}$ remain exact).

Note however that the model does not have interactions such as the ones that allow the ordinary neutral pion to decay into two photons: such effects are produced by the gauged Wess-Zumino-Witten [50, 51] Lagrangian, which is absent for this form of DM because it is assumed neutral under the SM local symmetries.

\subsection{Conserved currents}

The Lagrangian (2.9) is invariant under the global transformations

$$
\Sigma \rightarrow V^{\dagger} \Sigma V ; \quad V \in \mathrm{SU}(N)
$$

which give rise to a set of conserved Noetherian currents

$$
J_{b}^{\mu}=\left(1+\lambda_{h} \frac{|\phi|^{2}-v^{2}}{f^{2}}\right) \partial^{\mu} \pi_{d} g_{a d} \pi_{c} f_{b c a}-\frac{2}{f^{2}} B^{\mu \nu} f_{b c a} \pi_{c} g_{a e} f_{e d f} \partial_{\nu} \pi_{d} \mathbf{I m}\left(\lambda_{V} \operatorname{tr}\left\{T^{f} \Sigma^{\dagger}\right\}\right)
$$

Ignoring the interactions with the SM the canonical momentum are $\wp_{a}=g_{a b} \dot{\pi}_{a}$ in terms of which the charges (again ignoring the SM interactions) become

$$
Q_{b}=\int d^{3} \mathbf{x} J_{b}^{0}=\int d^{3} \mathbf{x} \pi_{c} f_{b c a} \wp_{a}
$$

and (ignoring possible sigma terms and other anomalies [52]) satisfy the algebra

$$
\left[Q_{a}, Q_{b}\right]=i f_{a b c} Q_{c}
$$

as expected.

The number of commuting conserved charges equals the rank of the group, which, in a Cartan basis, can be conveniently chosen as those associated with the $\pi_{i}$ :

$$
\left[Q_{i}, Q_{j}\right]=0 ; \quad Q_{i}=\sum_{\boldsymbol{\alpha}} \alpha_{i} \int d^{3} \mathbf{x} \pi_{\boldsymbol{\alpha}} \wp_{\boldsymbol{\alpha}}
$$

Assuming that these relations do not exhibit commutator anomalies [52] the charges $Q_{i}$ will be conserved; in particular this property will be reflected in the Boltzmann equations. It follows from the expression for $Q_{i}$ that the $\pi_{i}$ carry no charge, while $\pi_{ \pm \boldsymbol{\alpha}}$ carry opposite $i$-charges when $\alpha_{i} \neq 0$. 


\subsection{Parameters of the model}

The model we consider has then 4 parameters: the DMP mass $M$, the DMP decay constant $f$, the coupling constant of the DMP to the Higgs $\lambda_{h}$, and $\lambda_{V}$, the coupling constant of the DMP to the hypercharge vector field $B$ (from which follow the coupling to the $Z$ boson and the photon).

In the calculations below we will take $\lambda_{V}$ coupling to be real with magnitude

$$
\lambda_{V}=0.63 .
$$

We will see later that as far as the Boltzmann equations are concerned, any change in $\lambda_{V}$ can be absorbed in a redefinition of the other parameters (cf. the end of section 5), so this choice does not represent a loss of generality and is made for computational ease only. It is worth noting that according to naive dimensional analysis (NDA) [53] its value is $\lambda_{V} \sim$ $g^{\prime} /(4 \pi)^{2} \simeq 0.0023$, where $g^{\prime}$ is the $\mathrm{U}(1)_{Y}$ gauge coupling constant in the Standard Model.

For the rest of the parameters we impose just some loose constraints. We require that

$$
\lambda_{h}<1
$$

in order to ensure the model remain perturbative. ${ }^{5}$ We will see later that all the experimental constraints on the model also have simple scaling dependence on the couplings $\lambda_{h}$ (see section 6.1), so this constraint will also not restrict the generality of our results.

Since we assume that the DMP are the pseudo-Goldstone bosons of some underlying theory and are generated by the breaking of $\mathcal{G}_{\mathrm{DM}}$ to $\mathcal{H}_{\mathrm{DM}}$ at some scale $\Lambda$, consistency of the resulting chiral model requires [41]

$$
4 \pi f \gg M .
$$

For large values of $N$ the left hand side is expected to be suppressed by a factor of $1 / \sqrt{N}[54$, 55], which we do not include because we will restrict ourselves to low values of $N$.

Another constraint can be derived by requiring loop corrections not to dominate over the tree-level terms. In particular this should hold for the radiative corrections generated by the term proportional to $\lambda_{V}$ in (2.16), which includes vertices of the form $\left(\lambda_{V} / f^{n+2}\right) Z_{\mu \nu} \partial^{\mu} \pi \partial^{\nu} \pi \pi^{n}$. Two such vertices will generate loop corrections to the $\partial^{\mu} \pi \partial_{\mu} \pi \pi^{k} / f^{k}$ vertex of the first term in (2.16):

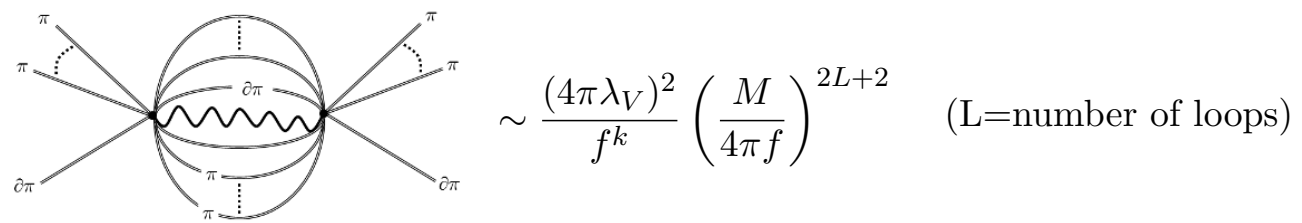

where we have assumed that all the terms in (2.16) that explicitly violate $\mathcal{G}_{\mathrm{DM}}$ are associated with the scale $M$, which we have used as an UV cutoff. We require (2.28) not to be larger

\footnotetext{
${ }^{5}$ In imposing this constraint we are being conservative as the perturbative unitarity limit is in fact $\lambda_{h}<4 \pi$.
} 


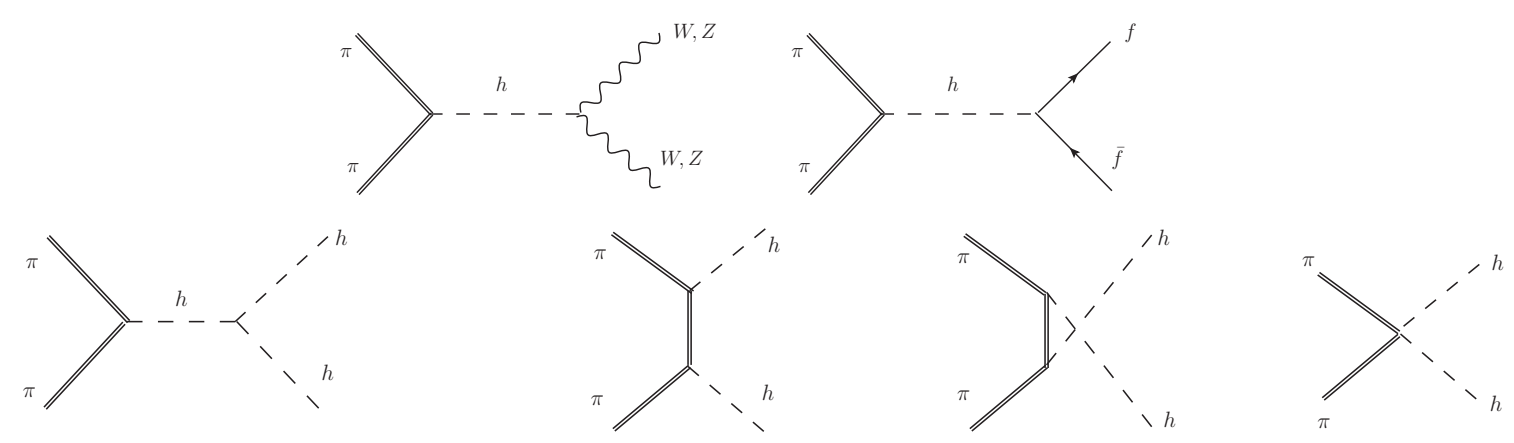

Figure 1. DMP $\rightarrow$ SM particle diagrams.

than the tree-level contribution, which implies (since $L$ can be arbitrarily large $)^{6}$

$$
f \geq\left[\max \left\{4 \pi \lambda_{V}, 1\right\}\right]^{1 / 2} \frac{M}{4 \pi} .
$$

\section{DMP interactions}

In this section we calculate the cross sections for the processes that dominate the Boltzmann equations that describe possible equilibration between the dark and SM sectors, and within the dark sector. The relevant interactions (2.16) separate into those that involve only DMP, and those that involve DMP and the SM scalar $\phi$ or the vector boson $B$. We also derive the reactions relevant for direct detection of the DMP. In all the calculations below we only consider $2 \rightarrow 2$ processes and will use the Cartan basis for the DMP.

\section{1 $\quad$ DMP $\rightarrow$ SM interactions}

There are two kinds of reactions:

Processes with only SM particles in the final state. These are of the form

$$
\begin{aligned}
\pi_{i} \pi_{i} & \rightarrow h^{*} \rightarrow \mathrm{SM}, \\
\pi_{\boldsymbol{\alpha}} \pi_{-\boldsymbol{\alpha}} & \rightarrow h^{*} \rightarrow \mathrm{SM}, \quad \pi_{i} \pi_{i} \rightarrow h h, \\
\pi_{\boldsymbol{\alpha}} \pi_{-\boldsymbol{\alpha}} & \rightarrow h h,
\end{aligned}
$$

for which the interaction terms in (2.16) are

$$
\mathcal{L}_{h-2 \pi}=\left(\frac{v \lambda_{h}}{\sqrt{2} f^{2}} h+\frac{\lambda_{h}}{4 f^{2}} h^{2}\right)\left[\sum_{i}\left(\partial \pi_{i}\right)^{2}+2 \sum_{\boldsymbol{\alpha}>0}\left|\partial \pi_{\boldsymbol{\alpha}}\right|^{2}\right],
$$

and the processes are shown in figure 1 .

\footnotetext{
${ }^{6}$ This can be refined by introducing the loop symmetry factor of $1 / \Gamma(L)$; the lower bound on $4 \pi f / M$ in terms of $x=4 \pi \lambda_{V}$ then becomes: $\sqrt{x}$ for $x>1 ; x^{1 / 3}$ for $1 \geq x \geq 1 / 8$, and below $x=0.125$ it is well approximated by $-(1 / \ln x)+[3 \ln (-\ln x)-\ln (2 \pi)] /\left[2(\ln x)^{2}\right]$. We will not, however, use these more complicated relations below.
} 
The cross sections for these processes are:

$$
\begin{aligned}
\sigma\left(\pi \pi \rightarrow W^{+} W^{-}\right)= & \frac{12 \kappa_{W}^{2}-4 \kappa_{W}+1}{4 \kappa_{\pi}^{2}} \beta_{W} \sigma_{\mathrm{SM}}, \\
\sigma(\pi \pi \rightarrow Z Z)= & \frac{12 \kappa_{Z}^{2}-4 \kappa_{Z}+1}{8 \kappa_{\pi}^{2}} \beta_{Z} \sigma_{\mathrm{SM}}, \\
\sigma(\pi \pi \rightarrow f \bar{f})= & \frac{\kappa_{f}}{2 \kappa_{\pi}^{2}} \beta_{f}^{3} \sigma_{\mathrm{SM}}, \\
\sigma(\pi \pi \rightarrow h h)= & \frac{s \lambda_{h}^{2}}{1024 \pi f^{4}} \frac{\beta_{h}}{\beta_{\pi}}\left\{\left[\frac{\left(1-2 \kappa_{\pi}\right)\left(1+\kappa_{h}\right)}{1-\kappa_{h}}-\frac{4 \lambda_{h} v^{2}}{f^{2}}\left(1-8 \kappa_{\pi}+2 \kappa_{h}\right)\right]^{2}\right. \\
& -\frac{4 \lambda_{h} v^{2}}{f^{2}}\left[\frac{\left(1-2 \kappa_{\pi}\right)\left(1+\kappa_{h}\right)}{1-\kappa_{h}}-\frac{4 \lambda_{h} v^{2}}{f^{2}} \frac{1-8 \kappa_{\pi}+4 \kappa_{\pi}^{2}+3 \kappa_{h}\left(4 \kappa_{\pi}-\kappa_{h}\right)}{1-2 \kappa_{h}}\right] \Upsilon \\
& \left.+\frac{16 \lambda_{h}^{2} v^{4}}{f^{4}}\left[\frac{2\left(\kappa_{h}-2 \kappa_{\pi}\right)^{4}}{\kappa_{\pi}+\kappa_{h}\left(\kappa_{h}-4 \kappa_{\pi}\right)}\right]\right\},
\end{aligned}
$$

where

$$
\begin{aligned}
\kappa_{i} & =m_{i}^{2} / s,\left(m_{\pi}=M\right) ; & \beta_{i} & =\sqrt{1-4 \kappa_{i}} ; \\
\sigma_{\mathrm{SM}} & =\frac{s \lambda_{h}^{2}}{16 \pi f^{4}} \frac{\kappa_{\pi}^{2}}{\beta_{\pi}} \frac{\left(1-2 \kappa_{\pi}\right)^{2}}{\left(1-\kappa_{h}\right)^{2}+\kappa_{h}\left(\Gamma_{h}^{2} / s\right)} ; & \Upsilon & =\frac{4\left(\kappa_{h}-2 \kappa_{\pi}\right)^{2}}{\beta_{\pi} \beta_{h}} \ln \left(\frac{1-2 \kappa_{h}+\beta_{\pi} \beta_{h}}{1-2 \kappa_{h}-\beta_{\pi} \beta_{h}}\right) .
\end{aligned}
$$

The overall factors of $s$ in $\sigma_{\mathrm{SM}}$ and $\sigma(\pi \pi \rightarrow h h)$ are a consequence of the derivative coupling of the DMP mandated by chiral symmetry; when small $s$ is allowed by the kinematics, this leads to a significant softening of the cross sections compared to the usual portal coupling of scalar DM to the SM. Other DMP couplings have a more complicated $s$ behavior (see below).

We neglected the Higgs width in the expression for $\sigma(\pi \pi \rightarrow h h)$ since it is never resonant (resonance occurs at $s \sim m_{h}^{2}$ while the reaction occurs only if $s>4 m_{h}^{2}$ ) and current data $[56,57]$ suggests $\Gamma_{h} \simeq \Gamma_{h}^{(\mathrm{SM})} \simeq 4 \mathrm{MeV}$ and $m_{h}=125 \mathrm{GeV}$ so that $\Gamma_{h}^{(\mathrm{SM})} / m_{h} \simeq$ $3.2 \times 10^{-6}$. For the $W, Z$ and $t$ reactions we can also ignore $\Gamma_{h}$ in $\sigma_{\mathrm{SM}}$ (defined in eq. (3.4)); the same is true for the other reactions if $M>m_{h} / 2$.

Processes involving DMP in the final state. These correspond to $\pi \pi \leftrightarrow \pi Z / \gamma$ for which the Lagrangian is given by

$$
\begin{aligned}
\mathcal{L}_{Z-3 \pi}=\frac{i \lambda_{V}}{f^{3}} i B^{\mu \nu}\{ & \sum_{\boldsymbol{\alpha}, \boldsymbol{\beta}} \partial_{\nu} \pi_{\boldsymbol{\alpha}} \partial_{\mu} \pi_{\boldsymbol{\beta}}^{\dagger} N_{\boldsymbol{\alpha},-\boldsymbol{\beta}} \pi_{\boldsymbol{\alpha}-\boldsymbol{\beta}}^{\dagger} \\
& \left.+\sum_{\boldsymbol{\alpha}}\left(\partial_{\nu} \pi_{\boldsymbol{\alpha}}\right)\left[2 \pi_{\boldsymbol{\alpha}}^{\dagger}\left(\partial_{\mu} \boldsymbol{\alpha} . \boldsymbol{\pi}\right)-2(\boldsymbol{\alpha} . \boldsymbol{\pi})\left(\partial_{\mu} \pi_{\boldsymbol{\alpha}}^{\dagger}\right)\right]\right\} .
\end{aligned}
$$

So there are 3 types of reactions (the first present only for $\mathrm{SU}(N), N>2$ ):

$$
\begin{array}{lllll}
\pi_{\boldsymbol{\alpha}}(p) & \pi_{\boldsymbol{\beta}}(q) & \leftrightarrow & \pi_{\boldsymbol{\alpha}+\boldsymbol{\beta}}(l) & V(k), \\
\pi_{\boldsymbol{\alpha}}(p) & \pi_{-\boldsymbol{\alpha}}(q) & \leftrightarrow & \pi_{i}(l) & V(k), \\
\pi_{\boldsymbol{\alpha}}(p) & \pi_{i}(q) & \leftrightarrow & \pi_{\boldsymbol{\alpha}}(l) & V(k)
\end{array}
$$



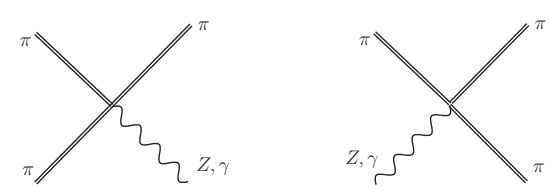

Figure 2. DMP scattering with $Z$ and $\gamma$.

( $V$ denotes $Z$ or $\gamma$ ), which are presented in figure 2. The cross sections are

$$
\begin{aligned}
& \sigma\left(\pi_{\boldsymbol{\alpha}} \pi_{\boldsymbol{\alpha}}^{\dagger} \rightarrow \pi_{i} V\right)=\sigma\left(\pi_{\boldsymbol{\alpha}} \pi_{i} \rightarrow \pi_{\boldsymbol{\alpha}} V\right)=\alpha_{i}^{2} \frac{K_{V}}{P} \sigma^{V}, \quad \sigma\left(\pi_{\boldsymbol{\alpha}} \pi_{\boldsymbol{\beta}} \rightarrow \pi_{\boldsymbol{\alpha}+\boldsymbol{\beta}} V\right)=\left|N_{\boldsymbol{\alpha}, \boldsymbol{\beta}}\right|^{2} \frac{K_{V}}{P} \sigma^{V}, \\
& \sigma\left(\pi_{i} V \rightarrow \pi_{\boldsymbol{\alpha}} \pi_{\boldsymbol{\alpha}}^{\dagger}\right)=\sigma\left(\pi_{\boldsymbol{\alpha}} V \rightarrow \pi_{\boldsymbol{\alpha}} \pi_{i}\right)=\frac{\alpha_{i}^{2}}{s_{V}} \frac{P}{K_{V}} \sigma^{V}, \quad \sigma\left(\pi_{\boldsymbol{\alpha}+\boldsymbol{\beta}} V \rightarrow \pi_{\boldsymbol{\alpha}} \pi_{\boldsymbol{\beta}}\right)=\frac{\left|N_{\boldsymbol{\alpha}, \boldsymbol{\beta}}\right|^{2}}{s_{V}} \frac{P}{K_{V}} \sigma^{V},
\end{aligned}
$$

where $s_{V}$ the number of spin degrees of freedom: $s_{Z}=3, s_{\gamma}=2$, and

$$
\begin{aligned}
\sigma^{Z} & =\left(\frac{3 s_{\mathrm{w}} \lambda_{V}}{f^{3}}\right)^{2} \frac{P^{2}}{16 \pi s}\left[\left(s-M^{2}-\frac{1}{3} m_{Z}^{2}\right)^{2}-\frac{4}{3}\left(s-\frac{4}{9} m_{Z}^{2}\right) K_{Z}^{2}\right], \\
\sigma^{\gamma} & =\left(\frac{3 c_{\mathrm{w}} \lambda_{V}}{f^{3}}\right)^{2} \frac{P^{2}}{24 \pi s}\left(s-M^{2}\right)^{2} .
\end{aligned}
$$

In the center of momentum $(\mathrm{CM})$ frame $K_{V}=|\mathbf{k}|=|\mathbf{1}|$ denotes the magnitude of the $V$ 3-momentum, and $P=|\mathbf{p}|=|\mathbf{q}|$ the magnitude of the 3-momentum of the pions not paired with the vector boson:

$$
K_{V}^{2}=\frac{\lambda\left(s, m_{V}^{2}, M^{2}\right)}{4 s}, \quad P^{2}=\frac{\lambda\left(s, M^{2}, M^{2}\right)}{4 s},
$$

with

$$
\lambda(a, b, c)=a^{2}+b^{2}+c^{2}-2 a b-2 b c-2 c a .
$$

\subsection{Direct-detection reaction}

The most important process that can contribute to the scattering of the DMP off heavy nuclei (relevant for direct DM detection [58-64]) is $\pi \psi \rightarrow \pi \psi$, where $\psi$ is SM fermion, and occurs through a $t$-channel $h$ exchange. The averaged amplitude-squared is

$$
\overline{|\mathcal{A}|^{2}}=\left(\frac{m_{\psi} \lambda_{h}}{2 f^{2}}\right)^{2}\left(\frac{t-2 M^{2}}{t-m_{h}^{2}}\right)^{2}\left(4 m_{\psi}^{2}-t\right),
$$

so that, in the CM frame, the corresponding cross section for this process is given by

$$
\begin{aligned}
\sigma(\pi \psi \rightarrow \pi \psi)= & \frac{1}{16 \pi s}\left(\frac{m_{\psi} \lambda_{h}}{2 f^{2}}\right)^{2}\left\{2\left(P^{2}-m_{h}^{2}+2 M^{2}+2 m_{\psi}^{2}\right)-\frac{\left(m_{h}^{2}-4 m_{\psi}^{2}\right)\left(m_{h}^{2}-2 M^{2}\right)^{2}}{m_{h}^{2}\left(m_{h}^{2}+4 P^{2}\right)}\right. \\
& \left.+\frac{\left(2 M^{2}+8 m_{\psi}^{2}-3 m_{h}^{2}\right)\left(2 M^{2}-m_{h}^{2}\right)}{4 P^{2}} \ln \left|\frac{4 P^{2}+m_{h}^{2}}{m_{h}^{2}}\right|\right\},
\end{aligned}
$$




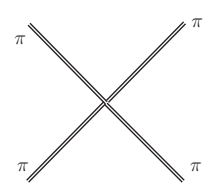

Figure 3. DMP $\rightarrow$ DMP scattering diagram.

where $P$ denotes the momentum of the incoming particles in the CM frame. When $M, m_{h} \gg P, m_{f}$ this cross section is approximated by

$$
\sigma(\pi \psi \rightarrow \pi \psi) \simeq \frac{1}{4 \pi s}\left(\frac{m_{\psi} \lambda_{h} M^{2}}{m_{h}^{2} f^{2}}\right)^{2}\left(m_{\psi}^{2}+\frac{P^{2}}{2}\right) \quad\left(M, m_{h} \gg P, m_{f}\right) .
$$

At low momentum transfer the effective interaction obtained from integrating the Higgs using (3.2) and the Standard Model $h \bar{f} f$ interaction $-\left(m_{\psi} / v\right) h \bar{\psi} \psi$ is

$$
\mathcal{L}_{\pi \pi \psi \psi}^{\text {(eff })}=-\left(\frac{\sqrt{2} m_{\psi} \lambda_{h} M^{2}}{m_{h}^{2} f^{2}}\right) \frac{1}{2} \pi^{2} \bar{\psi} \psi .
$$

\subsection{Pure DMP scattering}

Finally, we obtain the cross sections responsible for equilibrium within the DMP sector, $\pi \pi \rightarrow \pi \pi$, figure 3 . The lowest-order terms (taking $M$ real) in (2.16) are

$$
\mathcal{L}=\frac{1}{2}(\partial \boldsymbol{\pi})^{2}-\frac{1}{2} M^{2} \boldsymbol{\pi}^{2}+\frac{N}{16 f^{2}\left(N^{2}-2\right)}\left[\left(\partial \boldsymbol{\pi}^{2}\right)^{2}-\mu^{2}\left(\boldsymbol{\pi}^{2}\right)^{2}\right],
$$

where

$$
\mu^{2}=\frac{6 N^{2}-4}{N^{2}\left(N^{2}+1\right)} M^{2}
$$

and we have dropped terms that vanish on shell and will no contribute to the S-matrix.

In terms of DMP defined in the Cartan basis

$$
\boldsymbol{\pi}^{2}=\sum_{i} \pi_{i}^{2}+2 \sum_{\boldsymbol{\alpha}>0} \pi_{\boldsymbol{\alpha}} \pi_{-\boldsymbol{\alpha}} ; \quad \pi_{\boldsymbol{\alpha}}^{\dagger}=\pi_{-\boldsymbol{\alpha}},
$$

we have the following reactions:

\begin{tabular}{|llll|}
\hline reaction & \multicolumn{1}{c|}{ Lagrangian } & amplitude & crosssection \\
\hline$i i \rightarrow j j(i \neq j)$ & $-(u / 4) \pi_{i}^{2}\left(\square+\mu^{2}\right) \pi_{j}^{2}$ & $i u\left(s-\mu^{2}\right)$ & $\sigma_{0} / 2$ \\
$i i \rightarrow i i$ & $-(u / 8) \pi_{i}^{2}\left(\square+\mu^{2}\right) \pi_{i}^{2}$ & $i u\left(4 M^{2}-3 \mu^{2}\right)$ & $u^{2}\left(M^{2}-\frac{3}{4} \mu^{2}\right)^{2} /(2 \pi s)$ \\
$i i \rightarrow \boldsymbol{\alpha} \overline{\boldsymbol{\alpha}}$ & $-(u / 2) \pi_{i}^{2}\left(\square+\mu^{2}\right)\left|\pi_{\boldsymbol{\alpha}}\right|^{2}$ & $i u\left(s-\mu^{2}\right)$ & $\sigma_{0}$ \\
$\boldsymbol{\alpha} \overline{\boldsymbol{\alpha}} \rightarrow i i$ & $-(u / 2) \pi_{i}^{2}\left(\square+\mu^{2}\right)\left|\pi_{\boldsymbol{\alpha}}\right|^{2}$ & $i u\left(s-\mu^{2}\right)$ & $\sigma_{0} / 2$ \\
$\boldsymbol{\alpha} \overline{\boldsymbol{\alpha}} \rightarrow \boldsymbol{\beta} \overline{\boldsymbol{\beta}}(\boldsymbol{\alpha} \neq \boldsymbol{\beta})$ & $-u\left|\pi_{\boldsymbol{\beta}}\right|^{2}\left(\square+\mu^{2}\right)\left|\pi_{\boldsymbol{\alpha}}\right|^{2}$ & $i u\left(s-\mu^{2}\right)$ & $\sigma_{0}$ \\
$\boldsymbol{\alpha} \overline{\boldsymbol{\alpha}} \rightarrow \boldsymbol{\alpha} \overline{\boldsymbol{\alpha}}$ & $-(u / 2)\left|\pi_{\boldsymbol{\alpha}}\right|^{2}\left(\square+\mu^{2}\right)\left|\pi_{\boldsymbol{\alpha}}\right|^{2}$ & $2 i u\left(M^{2}-\mu^{2}\right)$ & $u^{2}\left(M^{2}-\mu^{2}\right)^{2} /(4 \pi s)$ \\
\hline
\end{tabular}

where $\overline{\boldsymbol{\alpha}}=-\boldsymbol{\alpha}, \overline{\boldsymbol{\beta}}=-\boldsymbol{\beta}$, and

$$
\sigma_{0}=\frac{u^{2}\left(s-\mu^{2}\right)^{2}}{16 \pi s}, \quad u=\frac{N}{2 f^{2}\left(N^{2}-2\right)} .
$$



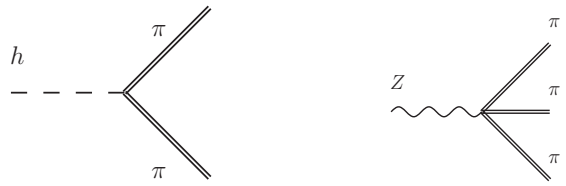

Figure 4. SM particle decays to DMP.

\subsection{Decays of SM particles to DMP}

Limits on the DMP parameters can be derived either from collider reactions or from potential deviations from SM decays. Reactions of the form $f \bar{f} \rightarrow \pi \pi$, where $f$ is a SM fermion, or $W$ fusion reactions $W W \rightarrow \pi \pi$, would mimic neutrino production at colliders. The limits, however, are very weak since these processes proceed through a virtual $h$ and so the amplitude will be proportional to small Yukawa coupling, or, for the case of heavy initial quarks, suppressed distribution functions.

The main limits are then derived form the two leading decay processes, figure 4, namely, $h \rightarrow \pi \pi$ and $Z \rightarrow \pi \pi \pi$, to which we now turn.

$\boldsymbol{h} \rightarrow \boldsymbol{\pi} \boldsymbol{\pi}$ decay. Using (2.16) and choosing a Hermitian $\pi$ basis we find that the width is given by

$$
\Gamma\left(h \rightarrow \pi_{a} \pi_{b}\right)=\Gamma_{h \pi \pi} \delta_{a b} ; \quad \Gamma_{h \pi \pi}=\frac{\left(\lambda_{h} v\right)^{2}}{16 \pi m_{h}}\left(\frac{m_{h}^{2}-2 M^{2}}{2 f^{2}}\right)^{2} \sqrt{1-\frac{4 M^{2}}{m_{h}^{2}}} \theta\left(m_{h}-2 M\right)
$$

in the Cartan basis $\Gamma\left(h \rightarrow \pi_{i} \pi_{i}\right)=\Gamma_{h \pi \pi}$ and $\Gamma\left(h \rightarrow \pi_{\boldsymbol{\alpha}} \pi_{-\boldsymbol{\alpha}}\right)=2 \Gamma_{h \pi \pi}$. Recent data [56, 57] favors a Higgs decay close to the SM prediction of $\sim 4 \mathrm{MeV}$ and a mass $m_{h} \sim 125 \mathrm{GeV}$; this requires $M>m_{h} / 2$, or $M<m_{h} / 2$ and $\Gamma_{h \pi \pi}<4 \mathrm{MeV}$, hence the constraint we use is

$$
f>5.9\left|\lambda_{h}\right|^{1 / 2}\left|7812.5-M^{2}\right|^{1 / 2}\left[1-\left(\frac{M}{62.5}\right)^{2}\right]^{1 / 8}, \quad M<62.5(M \text { in GeV }) .
$$

In the numerical solutions to Boltzmann equations for DMP for the $\mathrm{SU}(2)$ case (discussed below), we consider DMP masses in the interval $50 \mathrm{GeV} \leq M \leq 2000 \mathrm{GeV}$ so the $h \rightarrow \pi \boldsymbol{\pi}$ constraint plays an important role only for comparatively small values of $M$.

$\boldsymbol{Z} \rightarrow \boldsymbol{\pi} \boldsymbol{\pi} \boldsymbol{\pi}$ decay. The calculation is straightforward; using again a Hermitian DMP basis we find

$$
\Gamma(Z \rightarrow \pi \pi \pi)=\frac{M^{7} s_{\mathrm{w}}^{2} \lambda_{V}^{2}}{15\left(8 \pi f^{2}\right)^{3} r^{5 / 2}}\left(\sum_{a>b>c}\left|f_{a b c}\right|^{2}\right)\left[p_{E} \mathbf{E}(c)+p_{K} \mathbf{K}(c)\right]
$$


where $s_{\mathrm{w}}=\sin \theta_{\mathrm{w}}$, while $\mathbf{E}, \mathbf{K}$ denote the usual Elliptic functions, and

$$
\begin{aligned}
p_{E} & =\left(3 r^{8}+394 r^{6}-720 r^{4}+54 r^{2}-243\right), \\
p_{K} & =-\frac{1}{2}(r-1)^{3}\left(20 r^{6}+63 r^{5}+99 r^{4}+522 r^{3}+918 r^{2}+567 r+243\right), \\
c & =-\frac{(r-3)(1+r)^{3}}{16 r}, \\
r & =\frac{m_{Z}}{M} .
\end{aligned}
$$

For $\mathcal{H}_{\mathrm{DM}}=\mathrm{SU}(N)$ and our normalization conventions (2.11), (2.12) the summation involving the structure constants is given by

$$
\sum_{a>b>c}\left|f_{a b c}\right|^{2}=\frac{1}{3 !} \sum_{a, b, c}\left|f_{a b c}\right|^{2}=\frac{N\left(N^{2}-1\right)}{3} .
$$

Using the uncertainty in the invisible width of the $Z, \Gamma(Z)_{\text {inv }}$ we have limit

$$
\Gamma(Z \rightarrow \pi \pi \pi)<3 \times 10^{-3} \Gamma(Z)_{\mathrm{inv}}=3 \times 10^{-3} \frac{g^{2} m_{Z}}{32 \pi c_{\mathrm{w}}^{2}},
$$

which implies

$$
55.4>N\left(N^{2}-1\right)\left(\frac{m_{Z}^{3} \lambda_{V}}{f^{3}}\right)^{2} Q
$$

where

$$
Q=r^{-19 / 2}\left[p_{E} \mathbf{E}(u)+p_{K} \mathbf{K}(u)\right]
$$

The function $Q$ is monotonic; it vanishes as $r \rightarrow 3$ and approaches 0.75 as $r \rightarrow \infty$. Taking $N=2$, and $\lambda_{V}=0.63$, the most conservative limit (corresponding to taking $Q=3 / 4$ ) corresponds to

$$
f>51.43 \mathrm{GeV} \quad\left(M<\frac{m_{z}}{3}\right) .
$$

When $\lambda_{V}=0.063$, this limit becomes $f>23.87 \mathrm{GeV}$.

In the numerical analysis, we choose to work with DMP mass $\geq 50 \mathrm{GeV}$ and therefore the constraint from $Z \rightarrow \pi \pi \pi$ is of no importance.

\section{Thermal history of DMP}

We now turn to the derivation of the relic abundance of DMP. We follow the standard treatment (see e.g. [65]) and will consider only $2 \rightarrow 2$ processes.

\subsection{Boltzmann equations}

The change in the number density of particle of type $a$ due to collisions and the expansion of the universe is given by

$$
\begin{aligned}
\dot{n}_{a}+3 H n_{a} & =-\mathcal{C}_{a}, \\
\mathcal{C}_{a} & =\sum_{b, c, d} \int d \Phi\left|\mathcal{A}_{a+b \rightarrow c+d}\right|^{2}\left(f_{a} f_{b}-f_{c} f_{d}\right), \\
d \Phi & =d \Pi_{a} d \Pi_{b} d \Pi_{c} d \Pi_{d}(2 \pi)^{4} \delta^{(4)}\left(p_{a}+p_{b}-p_{c}-p_{d}\right),
\end{aligned}
$$


where $d \Pi$ denotes the phase-space volume

$$
d \Pi=\frac{g}{2 E_{\mathbf{p}}} \frac{d^{3} \mathbf{p}}{(2 \pi)^{3}},
$$

and $g$ is the number of internal degrees of freedom. The amplitude-squared $|\mathcal{A}|^{2}$ for the $a+b \rightarrow c+d$ process is understood to be averaged over initial and final states, and to include symmetry factors for identical particles in the final states. The functions $f$ are the particle phase-space distribution functions; the corresponding particle number density is

$$
n=g \int \frac{d^{3} \mathbf{p}}{(2 \pi)^{3}} f .
$$

We will assume that interactions are such that kinetic equilibrium is maintained [66]; we will also assume that particles densities are sufficiently small to ignore the effects of quantum statistics. In this case the energy dependence in the distribution functions is given by the Boltzmann factor: $f=\zeta \exp (-E / T)$. Since we are interested in the epoch when the DMP first decouple, all distribution functions will have the same temperature $T$; this will continue after decoupling provided no mass thresholds are crossed, or phase transitions occur.

The equilibrium distributions for a particle of mass $m$ is given by

$$
n^{(\mathrm{eq})}=g z \int \frac{d^{3} \mathbf{p}}{(2 \pi)^{3}} e^{-E / T}=\frac{z g m^{3}}{2 \pi^{2}} \frac{K_{2}(x)}{x}, \quad E=\sqrt{m^{2}+\mathbf{p}^{2}}, \quad x=\frac{m}{T}
$$

where $z$ is the fugacity in equilibrium. For the $\mathrm{SM} z_{\mathrm{SM}}=1$ to very good accuracy [67]; for the DMP, however, we will allow non-zero chemical potentials. Using the definition in (2.24) and the discussion below it, it follows that

$$
\mu_{j}^{(i)}=0, \quad \mu_{\boldsymbol{\alpha}}^{(i)}=-\mu_{-\boldsymbol{\alpha}}^{(i)},
$$

where $\mu_{a}^{(i)}$ denotes the chemical potential for particle $a$ associated with charge $Q_{i}$ so that $z \neq 1$ for those particles with non-zero conserved charges, as defined in section 2.1.

Substituting these definitions in the expression for $\mathcal{C}$ and using the standard definition of the scattering cross section $\sigma$ we find

$$
\begin{aligned}
\mathcal{C}_{a} & =\sum_{b, c, d}\left(\tilde{n}_{a} \tilde{n}_{b}-\frac{n_{a}^{(\mathrm{eq})} n_{b}^{(\mathrm{eq})}}{n_{c}^{(\mathrm{eq})} n_{d}^{(\mathrm{eq})}} \tilde{n}_{c} \tilde{n}_{d}\right)\langle\sigma v\rangle_{a+b \rightarrow c+d}, \\
\langle\sigma v\rangle_{a+b \rightarrow c+d} & =\frac{T g_{a} g_{b}}{2(2 \pi)^{4} n_{a}^{(\mathrm{eq})} n_{b}^{(\mathrm{eq})}} \int_{s_{o}}^{\infty} d s \frac{\lambda\left(s, m_{b}^{2}, m_{a}^{2}\right)}{\sqrt{s}} K_{1}(\sqrt{s} / T) \sigma_{a+b \rightarrow c+d}(s),
\end{aligned}
$$

where $\tilde{n}=z n, s=\left(p_{a}+p_{b}\right)^{2}=m_{a}^{2}+m_{b}^{2}+2 p_{a} \cdot p_{b}, \lambda(a, b, c)$ is defined in (3.10), and

$$
s_{o}=\max \left\{\left(m_{a}+m_{b}\right)^{2},\left(m_{c}+m_{d}\right)^{2}\right\} .
$$

In the definition of $s_{o}$ we used the condition (contained in the cross section) that $s$ should be large enough to create $c$ and $d$. 
For the pure DMP scattering processes that appear in the Boltzmann equations the averaged cross sections can be evaluated in closed form. We obtain, for example

$$
\begin{aligned}
\langle\sigma v\rangle_{\pi_{i} \pi_{i} \rightarrow \pi_{\alpha} \pi_{-\alpha}} & =\frac{1}{8} \frac{x}{z_{\pi_{a}} z_{\pi_{b}}\left[K_{2}(x)\right]^{2}} \frac{1}{M^{5}} \int_{4 M^{2}}^{\infty} d s \sqrt{s}\left(s-4 M^{2}\right) K_{1}(\sqrt{s} / T) \sigma_{0} \\
& =\frac{4 u^{2} M^{2}}{\pi} \frac{1}{z_{\pi_{a}} z_{\pi_{b}} x^{3}\left[K_{2}(x)\right]^{2}}\left[\frac{B x^{2}+3}{x} K_{2}(2 x)+\frac{B^{2} x^{2}+6}{4} K_{1}(2 x)\right],
\end{aligned}
$$

with similar expressions for the other relevant processes; in deriving this we used (3.18) and (3.19). For the relevant initial states $\left(\pi_{i} \pi_{i}\right.$ or $\left.\pi_{\boldsymbol{\alpha}} \pi_{-\boldsymbol{\alpha}}\right)$ we have $z_{i}=1=z_{\boldsymbol{\alpha}} z_{-\boldsymbol{\alpha}}$ so that in all cases of interest (see below) we can replace $z_{\pi_{a}} z_{\pi_{b}} \rightarrow 1$. Also $u$ is defined in (3.19), while $B$ is defined as

$$
B=1-\frac{\mu^{2}}{4 M^{2}}=\frac{N^{4}-\frac{1}{2} N^{2}+1}{N^{2}\left(N^{2}+1\right)}
$$

and $\mu$ is given in (3.15). In deriving the above result we used

$$
\int_{1}^{\infty} d y\left(y^{2}-1\right)^{n} K_{1}(2 x y)=\frac{n !}{2} \frac{K_{n}(2 x)}{x^{n+1}} .
$$

With the above preliminaries we can now find the relevant collision terms $\mathcal{C}_{a}$ (cf. eq. (4.1)) for the cases $a=\pi_{i}$ and $a=\pi_{\boldsymbol{\alpha}}$ that we abbreviate as $\mathcal{C}_{i}$ and $\mathcal{C}_{\boldsymbol{\alpha}}$ respectively. We will assume that all SM particles remain in equilibrium, so that $n_{\mathrm{SM}}=n_{\mathrm{SM}}^{(\mathrm{eq})}$. The tables of the relevant reactions (which do not cancel in $\mathcal{C}_{i, \alpha}$ ) are

\begin{tabular}{|c|c|}
\hline \multicolumn{2}{|c|}{$a=\pi_{\boldsymbol{\alpha}}$} \\
\hline$b$ & $c / d$ \\
\hline$\pi_{-\boldsymbol{\alpha}}$ & $W^{+} W^{-}, Z Z, f \bar{f}, h h, \pi_{j} V, \pi_{j} \pi_{j}, \pi_{\boldsymbol{\beta}} \pi_{-\boldsymbol{\beta}}$ \\
\hline$V$ & $\pi_{j} \pi_{\boldsymbol{\alpha}}, \pi_{\boldsymbol{\beta}} \pi_{\boldsymbol{\alpha}-\boldsymbol{\beta}}$ \\
\hline$\pi_{j}$ & $V \pi_{\boldsymbol{\alpha}}$ \\
\hline$\pi_{\boldsymbol{\beta}}$ & $V \pi_{\boldsymbol{\alpha}+\boldsymbol{\beta}}$ \\
\hline & $a=\pi_{i}$ \\
\hline$b$ & $c / d$ \\
\hline$\pi_{i}$ & $W^{+} W^{-}, Z Z, f \bar{f}, h h, \pi_{j} \pi_{j}, \pi_{\boldsymbol{\beta}} \pi_{-\boldsymbol{\beta}}$ \\
\hline$\pi_{\boldsymbol{\beta}}$ & $V \pi_{\boldsymbol{\beta}}$ \\
\hline
\end{tabular}

where $V$ represents $Z$ or $\gamma, \boldsymbol{\beta} \neq-\boldsymbol{\alpha}$, and a summation over $j$ and $\boldsymbol{\beta}$ is assumed.

Now, using (4.6) and noting that (4.5) implies

$$
\tilde{n}_{i}=n_{i}, \quad \tilde{n}_{\boldsymbol{\alpha}} \tilde{n}_{-\boldsymbol{\alpha}}=n_{\boldsymbol{\alpha}} n_{-\boldsymbol{\alpha}},
$$

and similarly for the equilibrium densities, we find

$$
\begin{aligned}
\mathcal{C}_{i}= & \left(n_{i}^{2}-n_{i}^{(\mathrm{eq}) 2}\right)\langle\sigma v\rangle_{\pi_{i} \pi_{i} \rightarrow \mathrm{SM}}+\sum_{\boldsymbol{\alpha}} \tilde{n}_{\boldsymbol{\alpha}}\left(n_{i}-n_{i}^{(\mathrm{eq})}\right)\left[\langle\sigma v\rangle_{\pi_{i} \pi_{\boldsymbol{\alpha}} \rightarrow \gamma \pi_{\boldsymbol{\alpha}}}+\langle\sigma v\rangle_{\pi_{i} \pi_{\boldsymbol{\alpha}} \rightarrow Z \pi_{\boldsymbol{\alpha}}}\right] \\
& +\sum_{\boldsymbol{\alpha}} n_{i}^{(\mathrm{eq})}\left(\frac{n_{i}}{n_{i}^{(\mathrm{eq})}}-\frac{n_{\boldsymbol{\alpha}}}{n_{\boldsymbol{\alpha}}^{(\mathrm{eq})}} \frac{n_{-\boldsymbol{\alpha}}}{n_{-\boldsymbol{\alpha}}^{(\mathrm{eq})}}\right)\left[n_{Z}^{(\mathrm{eq})}\langle\sigma v\rangle_{\pi_{i} Z \rightarrow \pi_{\boldsymbol{\alpha}} \pi_{-\alpha}}+n_{\gamma}^{(\mathrm{eq})}\langle\sigma v\rangle_{\pi_{i} \gamma \rightarrow \pi_{\boldsymbol{\alpha}} \pi_{-\alpha}}\right] \\
& +\sum_{j \neq i}\left(n_{i}^{2}-\frac{n_{i}^{(\mathrm{eq}) 2}}{n_{j}^{(\mathrm{eq})} n_{2}} n_{j}^{2}\right)\langle\sigma v\rangle_{\pi_{i} \pi_{i} \rightarrow \pi_{j} \pi_{j}}+\sum_{\boldsymbol{\alpha}}\left(n_{i}^{2}-\frac{n_{i}^{(\mathrm{eq}) 2}}{n_{\boldsymbol{\alpha}}^{(\mathrm{eq})} n_{-\boldsymbol{\alpha}}^{(\mathrm{eq})}} n_{\boldsymbol{\alpha}} n_{-\boldsymbol{\alpha}}\right)\langle\sigma v\rangle_{\pi_{i} \pi_{i} \rightarrow \pi_{\boldsymbol{\alpha}} \pi_{-\alpha}}
\end{aligned}
$$


and

$$
\begin{aligned}
\mathcal{C}_{\boldsymbol{\alpha}}= & \left(n_{\boldsymbol{\alpha}} n_{-\boldsymbol{\alpha}}-n_{\boldsymbol{\alpha}}^{(\mathrm{eq})} n_{-\boldsymbol{\alpha}}^{(\mathrm{eq})}\right)\langle\sigma v\rangle_{\pi_{\boldsymbol{\alpha}} \pi_{-\boldsymbol{\alpha}} \rightarrow \mathrm{SM}} \\
& +\sum_{i}\left(n_{\boldsymbol{\alpha}} n_{-\boldsymbol{\alpha}}-n_{\boldsymbol{\alpha}}^{(\mathrm{eq})} n_{-\boldsymbol{\alpha}}^{(\mathrm{eq})} \frac{n_{i}}{n_{i}^{(\mathrm{eq})}}\right)\left[\langle\sigma v\rangle_{\pi_{\boldsymbol{\alpha}} \pi_{-\boldsymbol{\alpha}} \rightarrow \pi_{i} \gamma}+\langle\sigma v\rangle_{\pi_{\boldsymbol{\alpha}} \pi_{-\alpha} \rightarrow \pi_{i} Z}\right] \\
& +\sum_{i}\left(n_{\boldsymbol{\alpha}} n_{-\boldsymbol{\alpha}}-\frac{n_{\boldsymbol{\alpha}}^{(\mathrm{eq})} n_{-\boldsymbol{\alpha}}^{(\mathrm{eq})}}{n_{i}^{(\mathrm{eq})} n_{i}^{2}}\right)\langle\sigma v\rangle_{\pi_{\boldsymbol{\alpha}} \pi_{-\boldsymbol{\alpha}} \rightarrow \pi_{i} \pi_{i}} \\
& +\sum_{\boldsymbol{\beta} \neq \pm \boldsymbol{\alpha}}\left(n_{\boldsymbol{\alpha}} n_{-\boldsymbol{\alpha}}-\frac{n_{\boldsymbol{\alpha}}^{(\mathrm{eq})} n_{-\boldsymbol{\alpha}}^{(\mathrm{eq})}}{n_{\boldsymbol{\beta}}^{(\mathrm{eq})} n_{-\boldsymbol{\beta}}^{(\mathrm{eq})}} n_{\boldsymbol{\beta}} n_{-\boldsymbol{\beta}}\right)\langle\sigma v\rangle_{\pi_{\boldsymbol{\alpha}} \pi_{-\boldsymbol{\alpha}} \rightarrow \pi_{\boldsymbol{\beta}} \pi_{-\boldsymbol{\beta}}},
\end{aligned}
$$

where the contributions coming from $\pi_{\boldsymbol{\alpha}} V \rightarrow \pi_{i} \pi_{\boldsymbol{\alpha}}(V=Z, \gamma)$ and $\pi_{\boldsymbol{\alpha}} \pi_{i} \rightarrow V \pi_{\boldsymbol{\alpha}}$ cancel, as do those from $\pi_{\boldsymbol{\alpha}} \pi_{\boldsymbol{\beta}} \rightarrow V \pi_{\boldsymbol{\alpha}+\boldsymbol{\beta}}$ and $\pi_{\boldsymbol{\alpha}} V \rightarrow \pi_{\boldsymbol{\beta}} \pi_{\boldsymbol{\alpha}-\boldsymbol{\beta}}$. We have also defined, using (4.6),

$$
\begin{aligned}
\langle\sigma v\rangle_{\pi_{i} \pi_{i} \rightarrow \mathrm{SM}}= & \langle\sigma v\rangle_{\pi_{i} \pi_{i} \rightarrow W W}+\langle\sigma v\rangle_{\pi_{i} \pi_{i} \rightarrow Z Z}+\sum_{f}\langle\sigma v\rangle_{\pi_{i} \pi_{i} \rightarrow f f}+\langle\sigma v\rangle_{\pi_{i} \pi_{i} \rightarrow h h} \\
= & \frac{T}{32 \pi^{4} n_{i}^{(\mathrm{eq})} 2} \int_{0}^{\infty} d s s^{3 / 2} K_{1}(\sqrt{s} / T) \beta_{\pi}^{2}\left[\sigma_{\pi_{i} \pi_{i} \rightarrow W W}+\sigma_{\pi_{i} \pi_{i} \rightarrow Z Z}\right. \\
& \left.+\sum_{f} \sigma_{\pi_{i} \pi_{i} \rightarrow f \bar{f}}+\sigma_{\pi_{i} \pi_{i} \rightarrow h h}\right]
\end{aligned}
$$

and similarly for $\pi_{\alpha} \pi_{-\alpha} \rightarrow$ SM.

\subsection{Contributions from SM $\rightarrow$ DMP decays}

The effects of Higgs decays into DMP, when kinematically allowed, can be included in the Boltzmann equation in two equivalent ways. We can include them in the total $h$ width:

$$
\Gamma_{h}=\Gamma_{h}^{\mathrm{SM}}+\Gamma(h \rightarrow \pi \pi)
$$

and use this expression in the cross sections involving Higgs exchange. Or, alternatively, we can exclude these effects from the Higgs propagators (see e.g. [68]):

$$
\frac{m_{h} \Gamma_{h}}{\left(s-m_{h}^{2}\right)^{2}+m_{h}^{2} \Gamma_{h}^{2}} \rightarrow \frac{m_{h} \Gamma_{h}}{\left(s-m_{h}^{2}\right)^{2}+m_{h}^{2} \Gamma_{h}^{2}}-\pi \delta\left(s-m_{h}^{2}\right) \Theta\left(s-4 m_{i}^{2}\right)
$$

and include them in suitable additions $\mathcal{C}_{i, \alpha}^{\text {(decay) }}$ to the collision terms; explicitly (see appendix A)

$$
\mathcal{C}_{i}^{\text {(decay) }}=N_{H}^{i} n_{H}^{(\mathrm{eq})} \frac{K_{1}\left(x_{H}\right)}{K_{2}\left(x_{H}\right)} \Gamma(h \rightarrow \pi \pi)
$$

where $N_{h}^{(i)}$ counts the number of produced $\pi_{i}: N_{h}^{(i)}=2$ ! for $h \rightarrow \pi_{i} \pi_{i}$ and $N_{h}^{(i)}=1$ for $h \rightarrow \pi_{\boldsymbol{\alpha}} \pi_{-\boldsymbol{\alpha}} ; \Gamma(h \rightarrow \pi \pi)$ is given in (3.20), and $x_{i}=m_{i} / T$. An analogous equation holds for $\mathcal{C}_{\alpha}^{\text {(decay) }}$.

If we assume that the recently observed particle at the LHC $[56,57]$ is the SM Higgs, it's very small total width ensures that the effects from Higgs decay to DMP are negligible. We have checked that for realistic DMP masses the contribution of $Z \rightarrow \pi \pi \pi$ decays in the Boltzmann equations (see appendix A) are also negligible. 


\section{Solving the Boltzmann equations for the SU(2) case}

The simplest non-trivial group is $\mathcal{H}_{\mathrm{DM}}=\mathrm{SU}(2)$, which we consider as an illustrative example of the formalism; the same approach can be used for any $N$, though with the calculations become increasingly cumbersome. For $N=2$ there is a single conserved charge and 3 DMP states that we label as $o, \pm$, with the first associated with the Cartan generator.

As usually we find it convenient to rewrite the Boltzmann equations (BE) (4.1), (4.13), (4.14) by defining

$$
x=\frac{M}{T}, \quad Y_{r}=\frac{1}{s} n_{r}, \quad Y_{r}^{(\mathrm{eq})}=\frac{1}{s} n_{r}^{(\mathrm{eq})},
$$

where $T$ denotes the photon temperature and $s$ the entropy density:

$$
s=\frac{2 \pi^{2}}{45} g_{s}(T) T^{3} ; \quad g_{s}(T)=\sum_{k} r_{k} g_{k}\left(\frac{T_{k}}{T}\right)^{3} \theta\left(T-m_{k}\right) ;
$$

here $k$ runs over all particles, $T_{k}$ is the temperature of particle $k$ and $g_{k}$ its number of internal degrees of freedom, and $r_{k}=1(7 / 8)$ when $k$ is a bosons (fermion). We will also make use of Friedman's equation,

$$
H^{2}=\frac{8 \pi G}{3} \rho=\frac{4 \pi^{3} G}{45} g(T) T^{4} ; \quad g(T)=\sum_{k} r_{k} g_{k}\left(\frac{T_{k}}{T}\right)^{4} \theta\left(T-m_{k}\right) .
$$

In the following we will take $T_{k}$ for all SM particles (assuming $T$ is above that of the $e^{+} e^{-}$ annihilation epoch), so that $g_{s}(T)=g(T)$; we use the expression for $g(T)$ in ref. [69]. The explicit form of the equilibrium distribution is

$$
Y_{r}^{(\mathrm{eq})}=\frac{45}{4 \pi^{4}} \frac{g_{r}}{g_{s}(T)} z_{r} x_{r}^{2} K_{2}\left(x_{r}\right) \stackrel{x_{r} \gg 1}{\longrightarrow} a_{r} z_{r} x_{r}^{3 / 2} e^{-x_{r}} ; \quad x_{r}=\frac{m_{r}}{T}, \quad a_{r}=\frac{45}{4 \pi^{4}} \sqrt{\frac{\pi}{2}} \frac{g_{r}}{g_{s}(T)}
$$

where $z_{r}$ is the fugacity for particle $r$ and $g_{r}$ the number of internal degrees of freedom.

We will also consider model parameters where the SM and DM sectors are in equilibrium for temperatures $T>T_{f}$, such that $T_{f}<M$, so that the region of interest is $x>1$ and the DMP will not contribute ${ }^{7}$ to the effective number of relativistic degrees of freedom $g(T)=g_{\mathrm{SM}}(T)$.

In terms of $Y$ the Boltzmann equations take the form

$$
\frac{d Y_{r}}{d x}=-\sqrt{\frac{\pi g(T)}{45 G}} \frac{M}{x^{2}} C_{r}(Y), \quad C_{r}(Y)=\frac{1}{s^{2}} \mathcal{C}_{r}, \quad(r=o, \pm)
$$

where the collision terms are

$$
\begin{aligned}
C_{o}(Y)= & \left(Y_{o}^{2}-Y_{o}^{(\mathrm{eq})^{2}}\right)\langle\sigma v\rangle_{\pi_{o} \pi_{o} \rightarrow \mathrm{SM}}+\left(Y_{o}^{2}-Y_{+} Y_{-}\right)\langle\sigma v\rangle_{\pi_{o} \pi_{o} \rightarrow \pi_{+} \pi_{-}} \\
& +\left[Y_{o} Y_{o}^{(\mathrm{eq})}-Y_{+} Y_{-}+\left(Y_{+}+Y_{-}\right)\left(Y_{o}-Y_{o}^{(\mathrm{eq})}\right)\right]\langle\sigma v\rangle_{\pi_{+} \pi_{-} \rightarrow \pi_{o} V},
\end{aligned}
$$

\footnotetext{
${ }^{7}$ If $T_{f}>M$ then the situation is more complicated, as the DMP will contribute to the relativistic degrees of freedom during their decoupling form the SM. For $T \leq T_{f}$ the DMP temperature is determined by entropy conservation: $s_{\pi}\left(T_{\pi}\right) R^{3}=s_{\pi}\left(T_{f}\right) R_{f}^{3}$ and is in general different form the photon temperature.
} 
and

$$
\begin{gathered}
C_{ \pm}(Y)=\left(Y_{+} Y_{-}-Y_{o}^{(\mathrm{eq})^{2}}\right)\langle\sigma v\rangle_{\pi_{o} \pi_{o} \rightarrow \mathrm{SM}}+\left(Y_{+} Y_{-}-Y_{o}^{2}\right)\langle\sigma v\rangle_{\pi_{+} \pi_{-} \rightarrow \pi_{o} \pi_{o}} \\
c q+\left(Y_{+} Y_{-}-Y_{o} Y_{o}^{(\mathrm{eq})}\right)\langle\sigma v\rangle_{\pi_{+} \pi_{-} \rightarrow \pi_{o} V}
\end{gathered}
$$

where we used $Y_{+}^{(\mathrm{eq})} Y_{-}^{(\mathrm{eq})}=Y_{o}^{(\mathrm{eq})^{2}}$, and also

$$
Y_{o}^{(\mathrm{eq})} Y_{Z / \gamma}^{(\mathrm{eq})}\langle\sigma v\rangle_{\pi_{o} Z / \gamma \rightarrow \pi_{+} \pi_{-}}=Y_{o}^{(\mathrm{eq})} Y_{ \pm}^{(\mathrm{eq})}\langle\sigma v\rangle_{\pi_{o} \pi_{ \pm} \rightarrow Z / \gamma \pi_{ \pm}}=Y_{o}^{(\mathrm{eq})^{2}}\langle\sigma v\rangle_{\pi_{+} \pi_{-} \rightarrow \pi_{o} Z / \gamma},
$$

and defined

$$
\langle\sigma v\rangle_{\pi_{+} \pi_{-} \rightarrow \pi_{o} V}=\langle\sigma v\rangle_{\pi_{+} \pi_{-} \rightarrow \pi_{o} \gamma}+\langle\sigma v\rangle_{\pi_{+} \pi_{-} \rightarrow \pi_{o} Z} .
$$

For the $\mathrm{SU}(2)$ case there is a single non-trivial chemical potential (4.5) and an associated conserved charge

$$
q=Y_{-}-Y_{+} .
$$

Using $q$, the two independent Boltzmann equations become

$$
\begin{aligned}
& \frac{d Y_{+}}{d x}=-\sqrt{\frac{\pi g(T)}{45 G} \frac{M}{x^{2}}\{} {\left[Y_{+}\left(Y_{+}+q\right)-Y_{o}^{(\mathrm{eq}) 2}\right]\langle\sigma v\rangle_{\pi_{o} \pi_{o} \rightarrow \mathrm{SM}}+\left[Y_{+}\left(Y_{+}+q\right)-Y_{o}^{2}\right]\langle\sigma v\rangle_{\pi_{+} \pi_{-} \rightarrow \pi_{o} \pi_{o}} } \\
&\left.+\left[Y_{+}\left(Y_{+}+q\right)-Y_{o} Y_{o}^{(\mathrm{eq})}\right]\langle\sigma v\rangle_{\pi_{+} \pi_{-} \rightarrow \pi_{o} V}\right\}, \\
& \frac{d Y_{o}}{d x}=-\sqrt{\frac{\pi g(T)}{45 G}} \frac{M}{x^{2}}\left\{\left(Y_{o}^{2}-Y_{o}^{(\mathrm{eq})^{2}}\right)\langle\sigma v\rangle_{\pi_{o} \pi_{o} \rightarrow \mathrm{SM}}+\left[Y_{o}^{2}-Y_{+}\left(Y_{+}+q\right)\right]\langle\sigma v\rangle_{\pi_{o} \pi_{o} \rightarrow \pi_{+} \pi_{-}}\right. \\
&\left.+\left[\left(2 Y_{+}+q\right)\left(Y_{o}-Y_{o}^{(\mathrm{eq})}\right)-Y_{+}\left(Y_{+}+q\right)+Y_{o} Y_{o}^{(\mathrm{eq})}\right]\langle\sigma v\rangle_{\pi_{+} \pi_{-} \rightarrow \pi_{o} V}\right\} .
\end{aligned}
$$

From (4.6) we find that

$$
\begin{aligned}
Y_{o}^{(\mathrm{eq})} Y_{Z / \gamma}^{(\mathrm{eq})}\langle\sigma v\rangle_{\pi_{o} Z / \gamma \rightarrow \pi_{+} \pi_{-}} & =\frac{T}{2\left[2 \pi^{2} s(T)\right]^{2}} \int_{s_{o}}^{\infty} d s P K_{V} \sqrt{s} K_{1}(\sqrt{s} / T) \sigma^{Z / \gamma}, \\
Y_{o}^{(\mathrm{eq}) 2}\langle\sigma v\rangle_{\pi_{o} \pi_{o} \rightarrow \mathrm{SM}} & =\frac{T}{2\left[2 \pi^{2} s(T)\right]^{2}} \int_{s_{o}}^{\infty} d s \sqrt{s} P^{2} K_{1}(\sqrt{s} / T) \sigma_{\pi_{o} \pi_{o} \rightarrow \mathrm{SM}},
\end{aligned}
$$

where $\sigma^{Z / \gamma}$ are given in (3.8) and $P, K_{V}$ are defined in (3.9).

The $\langle\sigma v\rangle$ are plotted in figure 5 for a representative parameter space point. The SM cross section is almost $x$-independent (corresponding to a predominance of $s$-wave scattering), while the $\gamma / Z$ cross section is proportional to $1 / x$, indicating a predominance of $p$-wave scattering. It is interesting to note that the DMP $\rightarrow \mathrm{DMP}$ cross section has an unusual $1 / \sqrt{x}$ behavior for large $x$ that results from all particles having the same mass and the amplitude being non-zero and finite at threshold, which for this model is a consequence of the chiral couplings of the DMPs. One can see, that $\langle\sigma v\rangle_{\pi \pi \rightarrow \mathrm{SM}}$ is much smaller than $\langle\sigma v\rangle_{\pi \pi \rightarrow \pi V}$ or $\langle\sigma v\rangle_{\pi \pi \rightarrow \pi \pi}$ for the particular choice of parameters. The relevance of $\langle\sigma v\rangle_{\pi \pi \rightarrow \mathrm{SM}}$ can be understood by referring to figure 6 where we compare $\langle\sigma v\rangle_{\pi \pi \rightarrow \mathrm{SM}}$ and $\langle\sigma v\rangle_{\pi \pi \rightarrow \pi V}$ at the decoupling temperature (the point at which the DMP particle density 


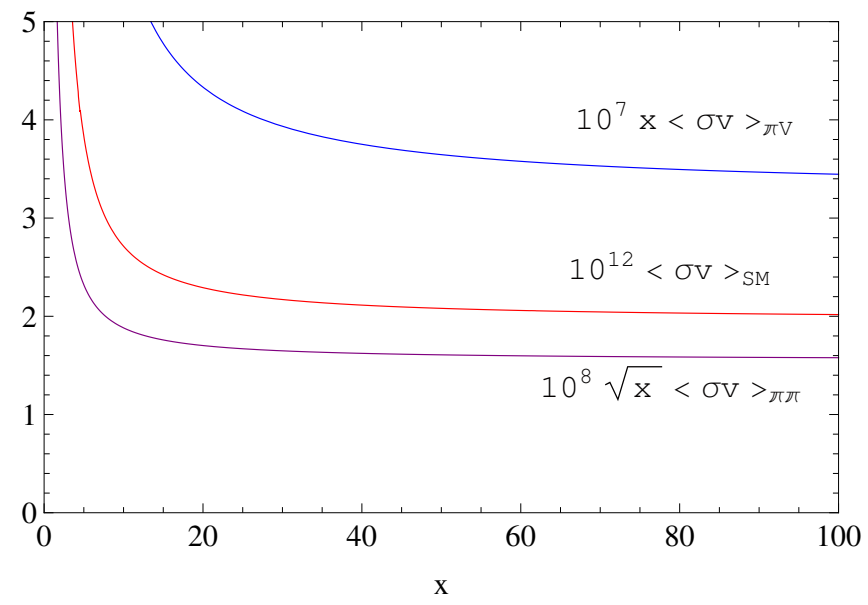

Figure 5. Cross sections for a representative set of parameters, $\left(M, f, \lambda_{h}, \lambda_{V}\right)=$ $(1000 \mathrm{GeV}, 950 \mathrm{GeV}, 0.01,0.63)$, for which the model satisfies the cold-dark matter and directdetection constraints. Top curve: $10^{7} x\langle\sigma v\rangle_{\pi \pi \rightarrow \pi V}$; middle curve: $10^{12}\langle\sigma v\rangle_{\pi \pi \rightarrow \mathrm{SM}}$; bottom curve: $10^{8} \sqrt{x}\langle\sigma v\rangle_{\pi \pi \rightarrow \pi \pi}$. The prefactors are chosen to fit the curves into the same graph and to illustrate the leading $x$ behavior. All the cross sections are in $\mathrm{GeV}^{-2}$.

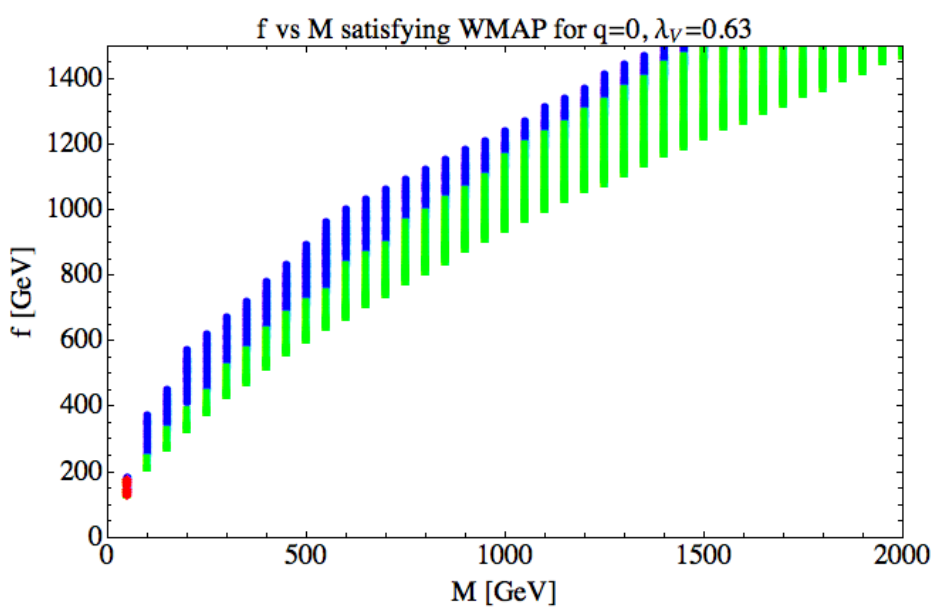

Figure 6. Region in the $M-f$ plane allowed by the CMD constraint (6.1) when $q=0, \lambda_{V}=0.63$, and $\left|\lambda_{h}\right|<1$. Blue points: subregion where $\langle\sigma v\rangle_{\pi \pi \rightarrow \mathrm{SM}}\left(x=x_{f}\right)>\langle\sigma v\rangle_{\pi \pi \rightarrow \pi V}\left(x=x_{f}\right)$. Green points: subregion where $\langle\sigma v\rangle_{\pi \pi \rightarrow \mathrm{SM}}\left(x=x_{f}\right)<\langle\sigma v\rangle_{\pi \pi \rightarrow \pi V}\left(x=x_{f}\right)$. Red points: subregion excluded by the Higgs decay constraint (3.21).

begins to deviate significantly from its equilibrium value - see section 5.1) for points that satisfy the cold-dark matter (CDM) relic-abundance constraint (see eq. (6.1) below).

To obtain the particle densities and their freeze out temperatures it is necessary to solve a system of coupled linear differential equations for $\left\{Y_{o}, Y_{+}\right\}$given by (5.11). The boundary conditions are determined by requiring that at low $x$ the DM sector is in equilibrium with the SM:

$$
x<x_{f}: \quad Y_{o}=Y_{o}^{(\mathrm{eq})}, \quad \text { and } \quad Y_{ \pm}=Y_{ \pm}^{(\mathrm{eq})}=\sqrt{Y_{o}^{(\mathrm{eq})} 2+\frac{q^{2}}{4}} \mp \frac{q}{2} .
$$


Note that (5.11) and (5.13) imply that both the equations and initial conditions are invariant under $Y_{+} \leftrightarrow Y_{-}$and $q \leftrightarrow-q$. The solutions to the equations (5.11) will be obtained numerically below; however the case of pure DM scattering can be solved analytically and is presented for completeness in appendix B.

For the following it is useful to note that $\langle\sigma v\rangle_{\pi_{o} \pi_{o} \rightarrow \mathrm{SM}}$ depends on $\lambda_{h}$ only in the combination $\lambda_{h} / f^{2}$, while $\langle\sigma v\rangle_{\pi_{+} \pi_{-} \rightarrow \pi_{o} V}$ depends on $\lambda_{V}$ only as $\lambda_{V} / f^{3}$. This implies that we can take $M, f$ and $\lambda_{h}$ as independent parameters, fixing $\lambda_{V}$ at some convenient value as in (2.25); any other value of $\lambda_{V}$ can be obtained by appropriate rescaling of $f$ and $\lambda_{h}$.

\subsection{Zero charge solutions}

When $q=0$ all DMP will have the same initial equilibrium distribution, the relevant solutions to the $\mathrm{BE}$ then correspond to $Y_{o, \pm}=Y$; substituting this (and $q=0$ ) in (5.11) we find

$$
\frac{d Y}{d x}=-\sqrt{\frac{\pi g(T)}{45 G}} \frac{M}{x^{2}}\left(Y-Y^{(\mathrm{eq})}\right)\left\{\left(Y+Y^{(\mathrm{eq})}\right)\langle\sigma v\rangle_{\pi_{o} \pi_{o} \rightarrow \mathrm{SM}}+Y\langle\sigma v\rangle_{\pi_{+} \pi_{-} \rightarrow \pi_{o} V}\right\},
$$

where we drop the $o, \pm$ subindices.

Approximate solutions to this equation are readily obtained. We find that to good accuracy (see figure 5) the cross sections have an $s$ and $p$ wave behaviors for $x>10$ :

$$
\langle\sigma v\rangle_{\pi_{o} \pi_{o} \rightarrow \mathrm{SM}} \simeq \sigma_{\mathrm{SM}}, \quad\langle\sigma v\rangle_{\pi_{+} \pi_{-} \rightarrow \pi_{o} V} \simeq \frac{1}{x} \sigma_{V},
$$

where $\sigma_{\mathrm{SM}, V}$ are approximately $x$-independent.

Near the decoupling temperature we write $Y=Y^{(\mathrm{eq})}+\Delta$ and neglect terms proportional to $d \Delta / d x$ and $\Delta^{2}$; then (5.14) becomes

$$
\Delta \simeq \frac{x^{2}}{2 \vartheta_{\mathrm{SM}}+\vartheta_{V} / x} ; \quad \vartheta_{\mathrm{SM}}=\sqrt{\frac{\pi g(T)}{45 G}} M \sigma_{\mathrm{SM}}, \quad \vartheta_{V}=\sqrt{\frac{\pi g(T)}{45 G}} M \sigma_{V},
$$

where we also approximated $d Y^{(\mathrm{eq})} / d x \simeq-Y^{(\mathrm{eq})}$.

For large $x$, in contrast, $\Delta \simeq Y \gg Y^{(\mathrm{eq})}$ and (5.14) becomes

$$
\frac{d \Delta}{d x}=-\frac{\vartheta_{\mathrm{SM}} x+\vartheta_{V}}{x^{3}} \Delta^{2} \Rightarrow \Delta_{\infty} \simeq \frac{x_{f}^{2}}{\vartheta_{\mathrm{SM}} x_{f}+\vartheta_{V} / 2},
$$

where $1 / \Delta\left(x_{f}\right)$ is neglected.

Finally the decoupling 'temperature' $x_{f}$ is obtained from the condition $\Delta\left(x_{f}\right)=$ $c Y^{(\mathrm{eq})}\left(x_{f}\right)$, where $c$ is a numerical constant. This gives

$$
\begin{aligned}
Y_{\infty} & =\frac{x_{f}^{2}}{\vartheta_{\mathrm{SM}} x_{f}+\vartheta_{V} / 2}, \\
x_{f} & =\ln \left[a c(c+2) \vartheta_{\mathrm{SM}} \xi^{-1 / 2}+a c(c+1) \vartheta_{V} \xi^{-3 / 2}\right] ; \quad \xi=\ln \left[c\left(\vartheta_{\mathrm{SM}}+\vartheta_{V}\right) a\right]
\end{aligned}
$$

where $a$ is defined in (5.4) and $\vartheta_{\mathrm{SM}}, \vartheta_{V}$ in (5.16); this result is better suited for the case $\vartheta_{V} \gg \vartheta_{\mathrm{SM}}$ than the one presented in [65]. We will follow this reference and choose $c(c+2)=$ 1 or, $c \simeq 0.414$. In calculating the relic abundance it is important to remember that $Y_{\infty}$ refers to each DMP species, so that the total abundance will be proportional to $3 Y_{\infty}$. 


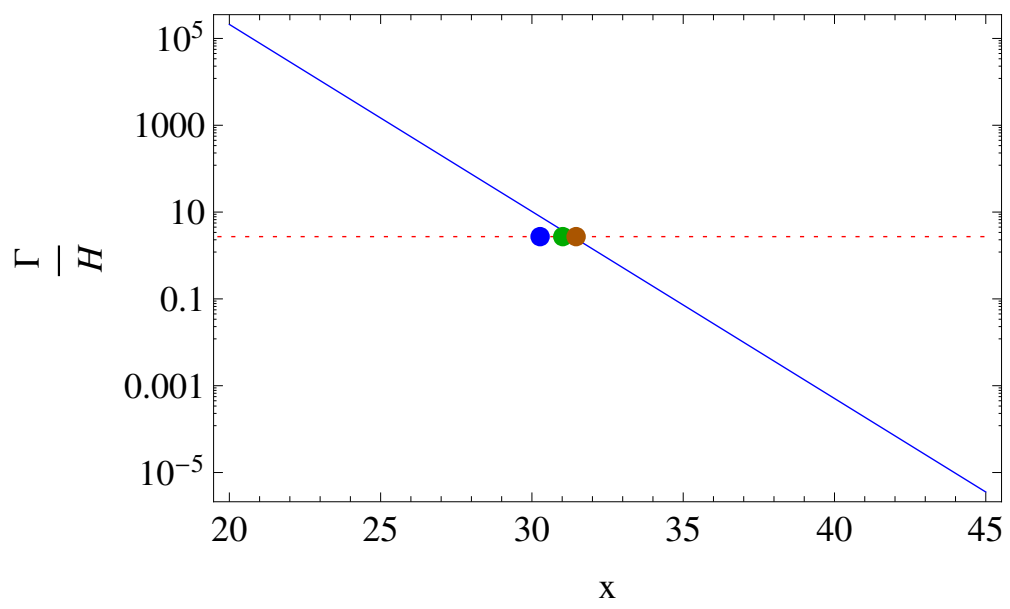

Figure 7. Plot of $\Gamma / H$ for the same parameters as in figure 5. We also include the values of $x_{f}$ obtained from the condition $\Delta=c Y^{(\mathrm{eq})}$ for $c=0.414,0.732,1$ (left, center and right heavy dots on the dashed line, respectively). The freeze-out condition $\Gamma=H$ corresponds to $x_{f} \simeq 31.3$ which coincides almost exactly with the $c=1$ value.

An alternative definition of $x_{f}$ can be derived by assuming $Y$ is close to $Y^{(\mathrm{eq})}$ and casting (5.14) in the form

$$
\frac{x}{Y_{o}^{(\mathrm{eq})}} \frac{d Y}{d x}=-\frac{\Gamma}{H}\left(\frac{Y}{Y_{o}^{(\mathrm{eq})}}-1\right) ; \quad \frac{\Gamma}{H}=\left(\frac{2 \vartheta_{\mathrm{SM}}+\vartheta_{V}}{x}\right) Y_{o}^{(\mathrm{eq})}
$$

so $x_{f}$ can be defined as the point where $\Gamma / H=1$. A plot of $\Gamma / H$ for representative values of the parameters, and a comparison with the previous definition of $x_{f}$ is given in figure 7 . This also illustrates that $x_{f}$ in general is large enough for the approximations (5.15) to be valid.

In figure 8 we compare the relic abundance derived numerically with the one obtained from (5.18), showing that, at least in this instance, the latter is reasonably accurate. From this figure one can also see that the decoupling point inferred from the numerical solutions equals the analytically obtained values within $10 \%$.

\subsection{Behavior for small values of $|q|$}

We now turn to the case where $q$ is small but non-vanishing. In this case it is convenient to define

$$
\begin{aligned}
& Y_{t}=Y_{o}+Y_{+}+Y_{-}=Y_{o}+2 Y_{+}+q, \\
& Y_{d}=\frac{Y_{+}+Y_{-}}{2}-Y_{o}=Y_{+}-Y_{o}+\frac{q}{2},
\end{aligned}
$$

in terms of which eqs. (5.11) become

$$
\begin{aligned}
& Y_{t}^{\prime}=-\frac{1}{3}\left(y_{t}^{2}+2 y_{d}^{2}\right)(A+B)+\left(y_{t}+y_{d}^{2}\right) B+\left[\frac{q^{2}}{4}(2 A+B)+3 A\right] \\
& Y_{d}^{\prime}=\frac{1}{3} y_{d}\left(y_{d}-2 y_{t}\right)\left(A+B+\frac{3}{2} C\right)-y_{d}\left(y_{d}+2\right) B+\frac{q^{2}}{4}\left(A+3 B h+\frac{3}{2} C\right),
\end{aligned}
$$




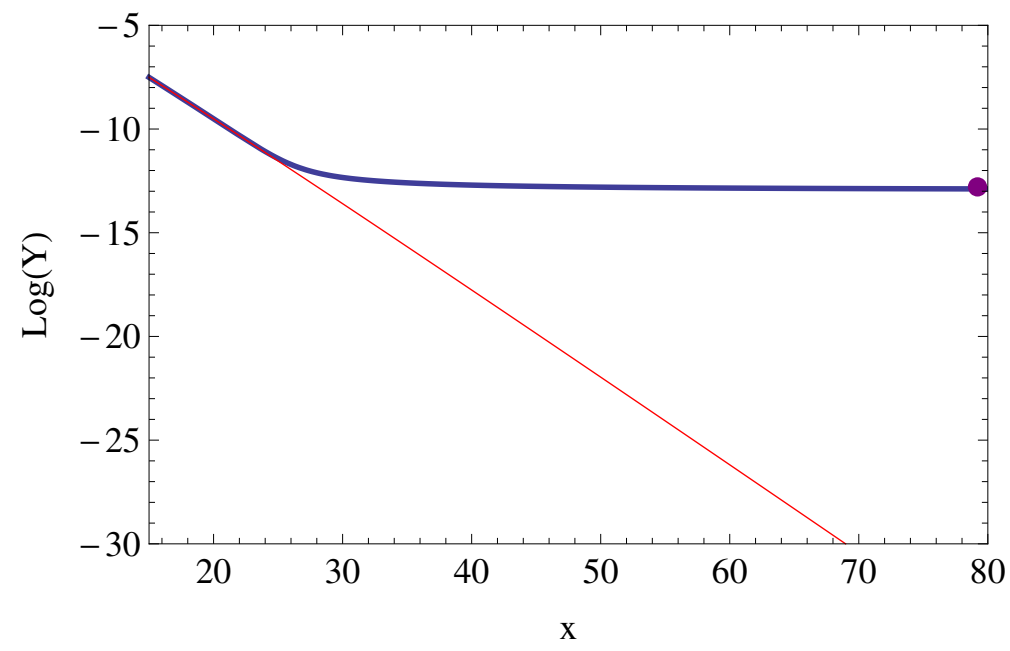

Figure 8. Plot of the yield $Y$ as a function $x$ for the representative point of figure 5 when $q=0$. Dark matter pion abundance is depicted in blue, and the equilibrium distribution is shown in red. The heavy dot on the right indicates the value of $Y_{\infty}$ obtained form (5.18) using $c=1$. All masses are in $\mathrm{GeV}$.

where $y_{t, d}=Y_{t, d} / Y_{o}^{(\mathrm{eq})}$ and

$$
\{A, B, C\}=\sqrt{\frac{\pi g(T)}{45 G}} \frac{M}{x^{2}} Y_{o}^{(\mathrm{eq}) 2}\left\{\langle\sigma v\rangle_{\pi_{o} \pi_{o} \rightarrow \mathrm{SM}},\langle\sigma v\rangle_{\pi_{+} \pi_{-} \rightarrow \pi_{o} V},\langle\sigma v\rangle_{\pi_{o} \pi_{o} \rightarrow \pi_{+} \pi_{-}}\right\}
$$

while the initial conditions (5.13) correspond to

$$
\begin{aligned}
& Y_{t}=Y_{t}^{(\mathrm{eq})}=Y_{o}^{(\mathrm{eq})}+2 \sqrt{Y_{o}^{(\mathrm{eq})^{2}}+q^{2} / 4} \\
& Y_{d}=Y_{d}^{(\mathrm{eq})}=-Y_{o}^{(\mathrm{eq})}+\sqrt{Y_{o}^{(\mathrm{eq})^{2}}+q^{2} / 4} .
\end{aligned}
$$

Now $Y_{t, d}$ are even in $q$, and assuming they are analytic in $q$ it follows that they depend on $q^{2}$; at $q=0$, we have $Y_{t}=3 Y$ and $Y_{d}=0$. Taking a derivative of (5.21) with respect to $q^{2}$ and evaluating at $q=0$ gives

$$
\begin{aligned}
& \left(\frac{\partial Y_{t}}{\partial q^{2}}\right)_{q=0}^{\prime}=-\frac{2 y}{Y_{o}^{(\mathrm{eq})}}\left(A+B-\frac{B}{2 y}\right)\left(\frac{\partial Y_{t}}{\partial q^{2}}\right)_{q=0}+\frac{2 A+B}{4} \\
& \left(\frac{\partial Y_{d}}{\partial q^{2}}\right)_{q=0}^{\prime}=-\frac{2 y}{Y_{o}^{(\mathrm{eq})}}\left(A+B+\frac{B}{y}+\frac{3}{2} C\right)\left(\frac{\partial Y_{d}}{\partial q^{2}}\right)_{q=0}+\frac{A+3 B+3 C / 2}{4}
\end{aligned}
$$

where $y=Y_{o} / Y_{o}^{(\mathrm{eq})}$. Initially,

$$
\begin{aligned}
& \left(\frac{\partial Y_{t}}{\partial q^{2}}\right)_{q=0}=\left(\frac{\partial Y_{t}^{(\mathrm{eq})}}{\partial q^{2}}\right)_{q=0}=\frac{1}{4 Y_{o}^{(\mathrm{eq})}}, \\
& \left(\frac{\partial Y_{d}}{\partial q^{2}}\right)_{q=0}=\left(\frac{\partial Y_{d}^{(\mathrm{eq})}}{\partial q^{2}}\right)_{q=0}=\frac{1}{8 Y_{o}^{(\mathrm{eq})}} .
\end{aligned}
$$


Now, a differential equation of the form

$$
Z^{\prime}=u Z+v
$$

has solution

$$
Z(x)=\int_{x_{i}}^{x} d s v(s) \exp \left[\int_{s}^{x} d r u(r)\right]+Z_{i} \exp \left[\int_{x_{i}}^{x} d r u(r)\right], \quad Z_{i}=\left(x_{i}\right) .
$$

In particular, if $v(x)>0$ for all $x$, and $Z_{i}>0$, then $Z(x)>0$ for $x>x_{i}$. Applying this to $Z=\left(\partial Y_{t, d} / \partial q^{2}\right)_{q=0}$, that have initial values $\sim 1 / Y_{o}^{(\mathrm{eq})}\left(x_{i}\right)>0$, we find that

$$
\left(\frac{\partial Y_{t, d}}{\partial q^{2}}\right)_{q=0}>0, \text { for } x \geq x_{i} .
$$

The relic abundance is obtained from the following expression [65]:

$$
\Omega_{\mathrm{DM}} h^{2}=2.7711 \times 10^{8}(M / \mathrm{GeV})\left(Y_{o}+Y_{+}+Y_{-}\right)_{x=\infty}=2.7711 \times\left. 10^{8}(M / \mathrm{GeV}) Y_{t}\right|_{x=\infty},
$$

since $Y_{t}(q \neq 0)>Y_{t}(q=0)$ (at least for small $q$ and with the other parameters fixed), it follows that

$$
\Omega_{\mathrm{DM}}\left(f, M, \lambda_{h}, \lambda_{V} ; q=0\right)<\Omega_{\mathrm{DM}}\left(f, M, \lambda_{h}, \lambda_{V} ; q \neq 0\right) .
$$

If $\Omega_{D M}\left(f, M, \lambda_{h}, \lambda_{V} ; q=0\right)<\Omega_{\mathrm{CDM}}$ for some parameters $\left\{f, M, \lambda_{h}, \lambda_{V}\right\}$, then there will be a non-zero $q$ such that $\Omega_{\mathrm{DM}}\left(f, M, \lambda_{h}, \lambda_{V} ; q\right)=\Omega_{\mathrm{CDM}}$. That is, if the predicted abundance falls below the observations when $q=0$, one can always "make-up" the difference by introducing an appropriate $q$ (at least when the difference is small). It follows that the the region in parameter space that can satisfy the CDM constraints is determined by

$$
\Omega_{D M}\left(f, M, \lambda_{h}, \lambda_{V} ; q=0\right)<\Omega_{\mathrm{CDM}} .
$$

A non-zero value of $q$ does not, of course, affect the direct-detection probability.

We illustrate Boltzmann equation solutions for small $q$ in figure 9. In general, there is a small range of $|q| \sim 10^{-12}-10^{-13}$ for which differences among the $Y_{+}, Y_{-}$and $Y_{0}$ abundances and between these and their equilibrium values are easily distinguished (it these cases the freeze-out temperatures for all three DMP components are very close). For smaller values, the effect of $q$ is negligible, while for larger values the effects of $q$ dominate the relic abundance and we find that $Y_{o}+Y_{+}+Y_{-} \simeq|q|$.

\section{$6 \quad$ Experimental limits on model parameters}

In this section we will consider the restrictions on the parameters of the model that are derived from cosmic background radiation using the data from the WMAP and PLANCK experiments $[6,7]$; we then consider direct detection constraints derived from the results obtained by the LUX [62], XENON100 and XENON1T experiments [63, 64]. We also include the consistency restrictions discussed in section 2.2. Brief comments on indirect detection are presented in Conclusions. 


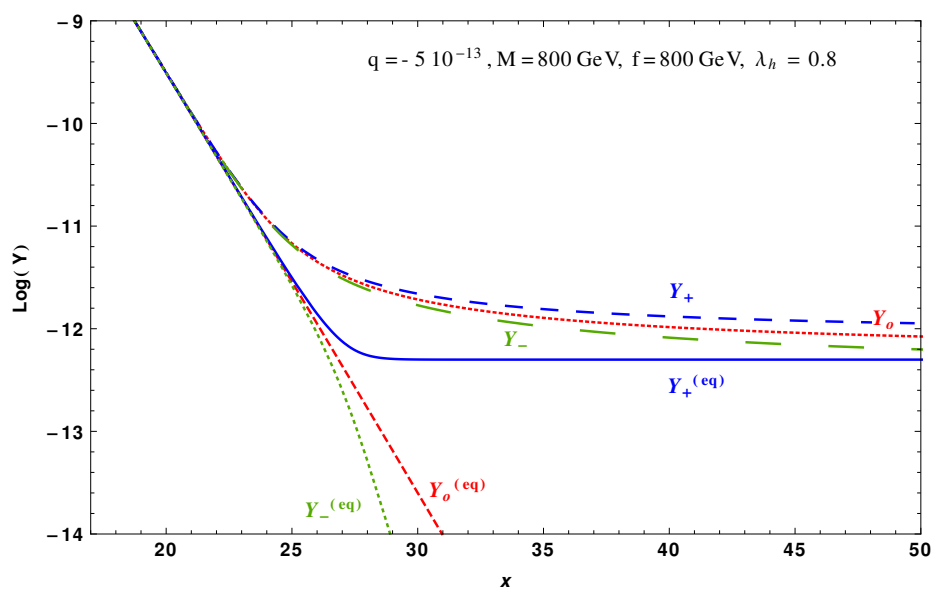

Figure 9. Illustration of the $q \neq 0$ case.

\subsection{Constraints from the cold dark matter (CDM) relic density measurements}

In this section we will obtain the numerical solution to the Boltzmann equations for the case $q=0$, when ${ }^{8} Y_{+}=Y_{-}=Y_{o}=Y$, and find the region of parameter space that meets the relic-abundance constraint $[70]^{9}$

$$
0.094 \leq \Omega_{\mathrm{DM}} h^{2} \leq 0.130
$$

As noted at the end of section 5 the solutions will depend on 3 independent parameters that we choose as $M, f$ and $\lambda_{h}$; without loss of generality, we fix $\lambda_{V}$ to the value (2.25). We scan the 3-dimensional parameter space $\left(M, f, \lambda_{h}\right)$ in the ranges $50 \mathrm{GeV} \leq M \leq 2 \mathrm{TeV}$, $50 \mathrm{GeV} \leq f \leq 1.5 \mathrm{TeV}, 10^{-4} \leq\left|\lambda_{h}\right| \leq 1$ for points allowed by (6.1); we also impose the constraint (2.27) and the one derived from $h \rightarrow \pi \pi$ decay, which is open in the low $M$ region (cf. section 3.4); note that in this region of parameter space the decay $Z \rightarrow \pi \pi \pi$ is kinematically forbidden, so that the restriction (3.26) does not apply. The $q \neq 0$ case is included by considering only the upper inequalities (see (5.31)). In particular, using $Y_{o}+Y_{+}+Y_{-} \simeq|q|$ for $q \gg 10^{-12}$ (cf. the end of section 5.2) we find that (5.31) satisfies (6.1) provided

$$
\frac{3.4 \times 10^{-10}}{M / \mathrm{GeV}}<|q|<\frac{4.7 \times 10^{-10}}{M / \mathrm{GeV}} \quad M \ll 100 \mathrm{GeV} .
$$

In figure 10 we plot the relic abundance $\Omega_{\mathrm{DM}} h^{2}$ and low-temperature distribution $Y_{\infty}$ as functions of $M$; note that (cf. the bottom panel of this figure) $Y_{\infty}$ has a non-trivial dependence on the DMP mass $M$. In figure 11 we show the region in the $M-f$ plane allowed by the CDM constraint (6.1) as well as the region allowed by $q \neq 0$. We see from figure 11 that, for fixed $\lambda_{V}, \Omega_{\mathrm{DM}}$ increases with $\lambda_{h}$ : and the region of sufficiently small (large) $\lambda_{h}$ corresponds to an under (over)-abundance of DM; this is in contrast to models where the

\footnotetext{
${ }^{8}$ Note that for $q=0$ case, DMP $\rightarrow$ DMP scattering cross sections do not enter eq. (5.14).

${ }^{9}$ The range we use corresponds to the WMAP results; the PLANCK constraints $0.112 \leq \Omega_{\mathrm{DM}} h^{2} \leq$ 0.128 [7], though more stringent, do not lead to significant changes in the allowed regions of parameter space.
} 

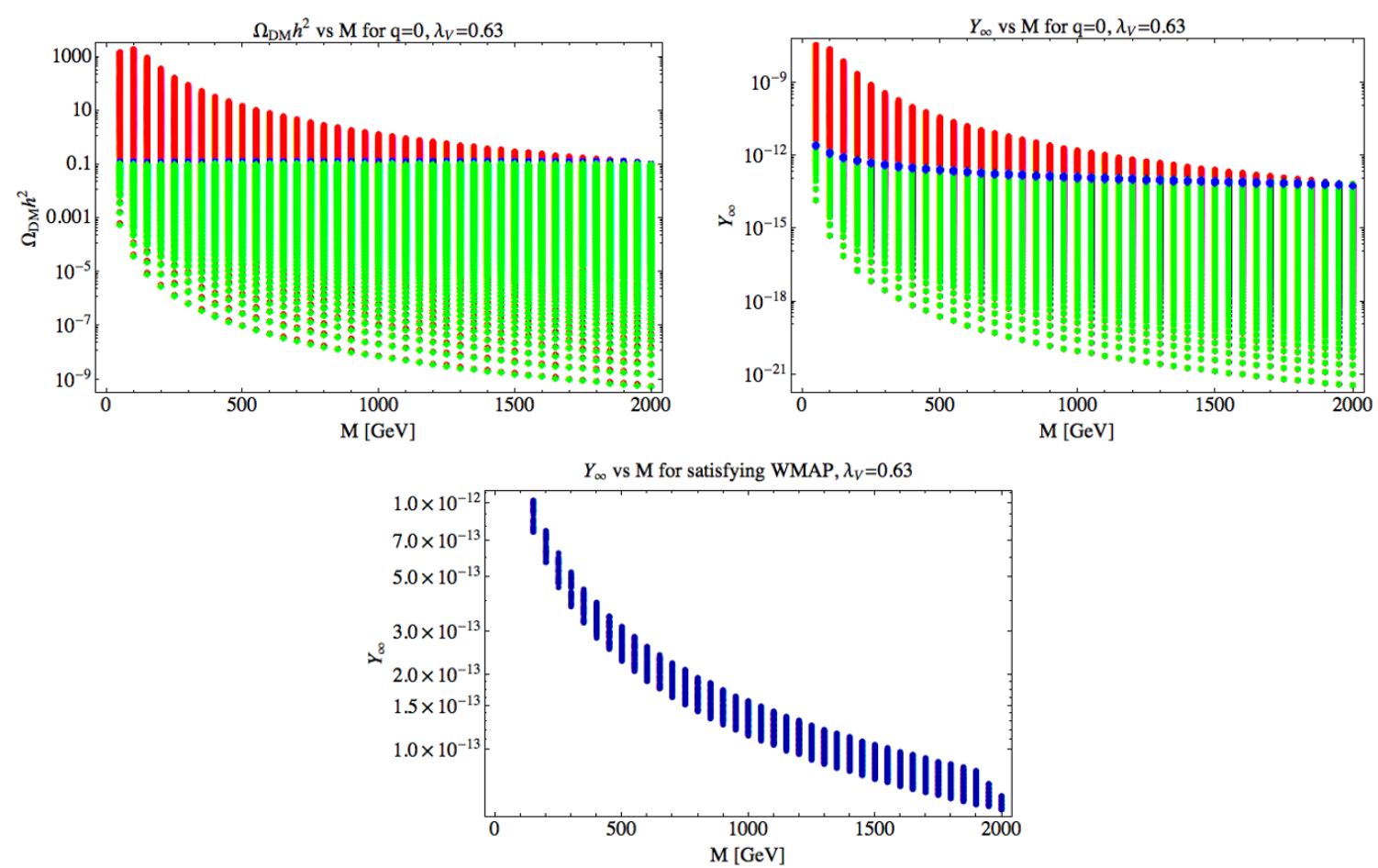

Figure 10. $\Omega_{\mathrm{DM}} h^{2}$ (top left) and $Y_{\infty}$ (top right) dependence on the DMP mass $M$ for all values of $f, \lambda_{h}$ in the region scanned, and when $q=0$ and $\lambda_{V}=0.63$. Red points: DM over-abundance $\left(\Omega_{\mathrm{DM}} h^{2}>0.13\right)$; blue points: region allowed by the CDM constraint (6.1); green points: DM underabundance $\left(\Omega_{\mathrm{DM}} h^{2}<0.094\right)$, which are allowed for appropriately chosen non zero $q$. The CDMallowed region for $Y_{\infty}$ is amplified in the bottom panel in order to better see the dependence on $M$.

leading coupling to the DM fields is through the Higgs-portal interaction [48, 49]. We trace this difference to the presence of the $\pi \pi \rightarrow Z \pi$ interaction, not to the derivative coupling: comparing figure 6 and figure 11 we see that the region where the relic abundance is small (but still allowed by the data) corresponds to small values of $\lambda_{h}$ and also to $\langle\sigma v\rangle_{\pi \pi \rightarrow \mathrm{SM}}(x=$ $\left.x_{f}\right)>\langle\sigma v\rangle_{\pi \pi \rightarrow \pi V}\left(x=x_{f}\right)$; while large values of $\lambda_{h}$ correspond to the larger allowed values of the relic abundance and to $\langle\sigma v\rangle_{\pi \pi \rightarrow \mathrm{SM}}\left(x=x_{f}\right)<\langle\sigma v\rangle_{\pi \pi \rightarrow \pi V}\left(x=x_{f}\right)$.

The allowed region in figure 11 corresponding to $q=0$ can be approximated analytically by

$$
39.65 \sqrt{M} \geq f \geq 9.33 M^{2 / 3} \quad\left(M, f \text { in } \mathrm{GeV} ; M<2 \mathrm{TeV},\left|\lambda_{h}\right| \leq 1, \lambda_{V}=0.63\right) .
$$

We now use this result to extend the CDM limits with reasonable accuracy to the whole region of parameter space of interest. To do that note first that the $s$-wave contribution to $\langle\sigma v\rangle_{\pi \pi \rightarrow \mathrm{SM}}$ is generated by the $\pi \pi \rightarrow h h$ contribution (cf. eq. (3.3)) so that in (5.15) $\sigma_{\mathrm{SM}} \sim\left(\lambda_{h} M / f^{2}\right)^{2}$ where the factor $\left(\left|\lambda_{h}\right| / f^{2}\right)^{2}$ comes from the vertices, while the factor of $M^{2}$ is needed to get the right units (the other mass scales can be ignored for $M>m_{h} / 2$ ) ${ }^{10}$ Similarly $\sigma_{V} \sim\left(\lambda_{V} M^{2} / f^{3}\right)^{2}$ where the factor $\left(\left|\lambda_{V}\right| / f^{3}\right)^{2}$ comes from the vertices, while the factor of $M^{4}$ is needed to get the right units.

\footnotetext{
${ }^{10}$ The factor of $M^{2}$ can also be seen to follow from the derivative couplings of the DMP; it typically leads to an over-abundance of DM for small $M$ and is excluded by the CMD data.
} 

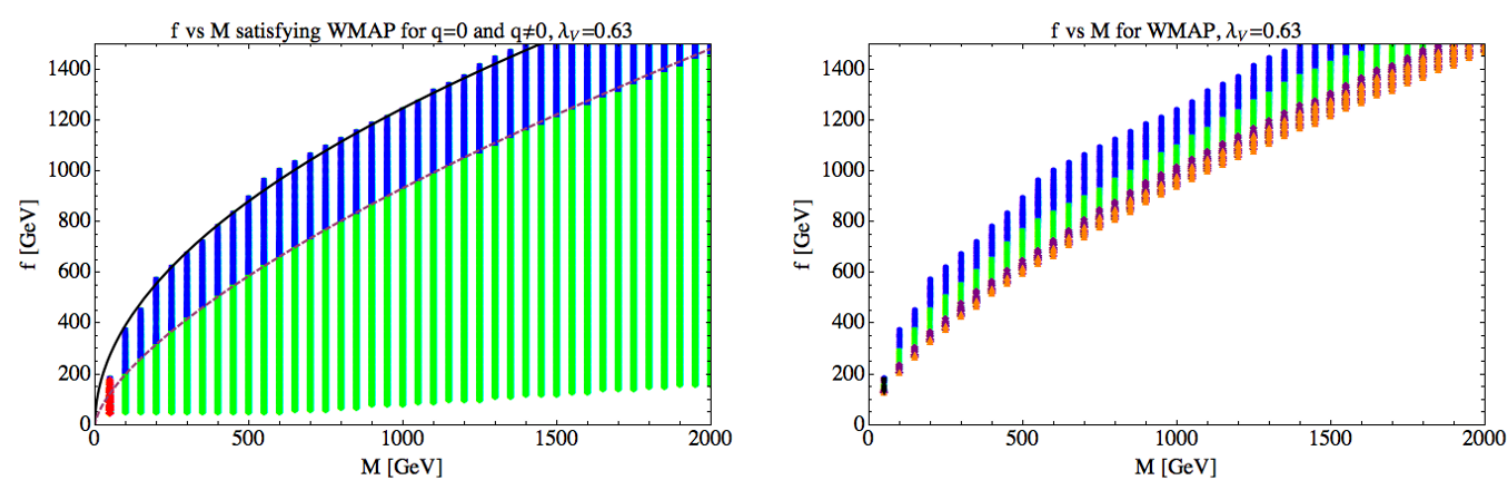

Figure 11. Left panel: region in the $f-M$ plane allowed by the CDM constraint (blue); the region corresponding to DM under-abundance (green); and the region excluded by the Higgs decay constraint eq. (3.21) (red). The solid and dashed black line correspond to the analytic approximations (6.3). Right panel: $\lambda_{h}$ dependence of the points in the region allowed by (6.1). Orange: $0.0001 \leq \lambda_{h} \leq 0.01$, purple: $0.01 \leq \lambda_{h} \leq 0.3$, green: $0.3 \leq \lambda_{h} \leq 0.6$, blue: $0.6 \leq \lambda_{h} \leq 1$. Red points are disallowed by (3.21).

Using this in (5.18) and (5.29) we find that up to a weak logarithmic dependence the parameters, $1 /\left(h^{2} \Omega_{D M}\right)$ will depend on a linear combination of $\left(\lambda_{h} M / f^{2}\right)^{2}$ and $\left(\lambda_{V} M^{2} / f^{3}\right)^{2}$. Comparing then figure 6 and figure 11 we find that the upper limit in (6.3) corresponds to parameters where $\sigma_{\mathrm{SM}}$ dominates and where the upper limit in (6.1) is saturated; while the lower limit in (6.3) corresponds to parameters where $\sigma_{V}$ dominates and where the lower limit in (6.1) is saturated. Using this in conjunction with (6.3) we find that the CDM constrain reduces to

$$
4.04 \times 10^{-7} \leq\left(\frac{\lambda_{h} M}{f^{2}}\right)^{2}+0.93\left(\frac{\lambda_{V} M^{2}}{f^{3}}\right)^{2} \leq 5.59 \times 10^{-7} \delta_{q 0} \quad(M, f \text { in } \mathrm{GeV}),
$$

where $\delta_{q 0}$ vanishes when $q \neq 0$ so that there is no upper limit in (6.4) in this case.

\subsection{Direct detection constraints}

The direct detection experiments probe the elastic scattering of DM particles off different kinds of materials [58-64]. For the present model the leading interaction is the $\pi N \rightarrow \pi N$ scattering of DMP off the material's nucleons $N$ (figure 12) through a $t$-channel Higgs exchange. The corresponding hard process was discussed in section 3.2 where we show that the DMP-quark scattering cross section (3.13) is proportional to $\left(\lambda_{h} M^{2} / f^{2}\right)^{2}$.

The parton-level interaction is converted to the nucleon level by using effective nucleon $f_{q}^{N}(N=p, n)$ couplings defined as [71]

$$
\left\langle N\left|m_{q} \bar{\psi}_{q} \psi_{q}\right| N\right\rangle=f_{q}{ }^{N} M_{N}
$$

where $M_{N}$ is the nucleon mass and $f_{u}^{p}=0.0160, f_{d}^{p}=0.0193, f_{s}^{p}=0.0410$, for the proton; $f_{u}^{n}=0.0108, f_{d}^{n}=0.0284, f_{s}^{n}=0.0409$ for the neutron; while for the heavy quarks the $f_{q}^{N}$ are generated by gluon exchange with the nucleon and are given by

$$
f_{Q}^{N}=\frac{2}{27}\left(1-\sum_{q=u, d, s} f_{q}^{N}\right) \quad Q=c, t, b .
$$




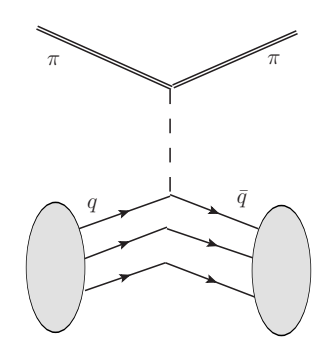

Figure 12. Direct detection process.

Then, DMP scattering with a nucleon composed of $Z$ protons and $A-Z$ neutrons is [71]

$$
\sigma_{\pi N}=\frac{1}{\pi}\left(\frac{m_{N}}{m_{N}+M}\right)^{2}\left(Z f^{p}+(A-Z) f^{n}\right)^{2} ; \quad \frac{f^{N}}{m_{N}}=\sum_{q} \frac{f_{q}^{N}}{m_{q}} \alpha_{q}
$$

and the sum is over all quarks. The $\alpha_{q}$ are effective couplings of DMP with the $q$-quarks, $\mathcal{L}=-\frac{1}{2} \alpha_{q} \overline{\psi_{q}} \psi q \pi \pi$ that can be read off (3.14):

$$
\alpha_{q}=\sqrt{2} \frac{m_{q} M^{2}}{m_{h}^{2}} \frac{\lambda_{h}}{f^{2}} .
$$

Using microOMEGAs [71] we evaluate numerically the DMP-nucleon scattering cross section for direct detection and then compare these results to the LUX, XENON100 and XENON1T bounds. The results are presented in figure 13. As indicated above, if $M$ is fixed the cross section depends only on $\lambda_{h} / f^{2}$ and, in fact, the direct detection bounds give rather simple expressions for the constraints on this ratio:

$$
\begin{array}{r}
\text { XENON100 }: f^{2} / \lambda_{h}>10^{5.5}, \\
\text { LUX }: f^{2} / \lambda_{h}>10^{6}, \\
\text { XENON1T }: f^{2} / \lambda_{h}>10^{6.5} .
\end{array}
$$

The corresponding restrictions on the $M-f$ plane over the CDM constrain are presented in figure 14.

\subsection{Combined constraints on DMP model}

The parameters in the model are constrained by the relations (2.29), (3.21), (6.4), and (6.9) that we collect here for convenience:

perturbativity : $f \geq \max \left\{\sqrt{4 \pi \lambda_{V}}, 1\right\} \frac{M}{4 \pi}$,

$$
\begin{aligned}
\text { Higgs decay : } & f>5.9\left|\lambda_{h}\right|^{1 / 2}\left|7812.5-M^{2}\right|^{1 / 2}\left[1-\left(\frac{M}{62.5}\right)^{2}\right]^{1 / 8} \quad(M<62.5[\mathrm{GeV}]), \\
\text { LUX : } & f>10^{3}\left|\lambda_{h}\right|^{1 / 2}, \\
\text { CDM : } & 4.04 \times 10^{-7} \leq\left(\frac{\lambda_{h} M}{f^{2}}\right)^{2}+0.93\left(\frac{\lambda_{V} M^{2}}{f^{3}}\right)^{2} \leq 5.59 \times 10^{-7} \delta_{q, 0}, \quad(6.10)
\end{aligned}
$$

where $f, M$ are in $\mathrm{GeV}$, and we used the LUX limit. The $\delta_{q, 0}$ factor indicates that the corresponding limit disappears when non-zero values of $q$ are allowed. 


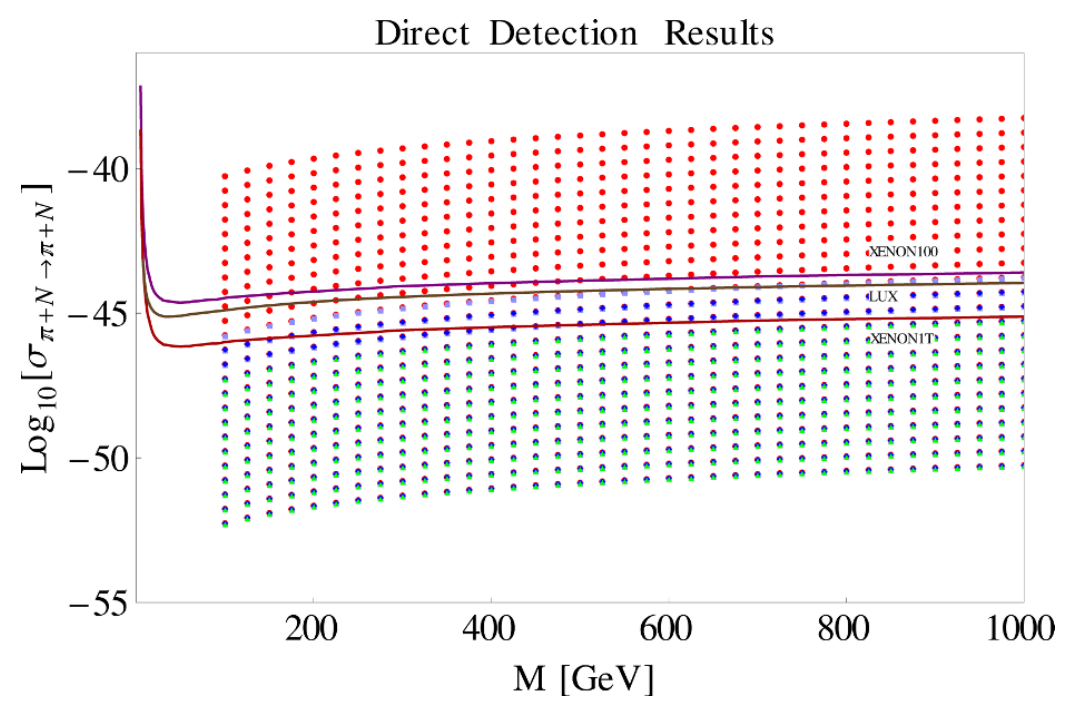

Figure 13. Direct detection constraints from the XENON and LUX experiments. XENON100 excludes all points above the solid line in purple at the top, which corresponds to the constraint $\lambda_{h} / f^{2}<10^{-5.5}$; the recent LUX results gives $\lambda_{h} / f^{2}<10^{-6}$. XENON1T is projected to exclude all points above the lower (red) solid line and would correspond to the constraint $\lambda_{h} / f^{2}<10^{-6.5}$.
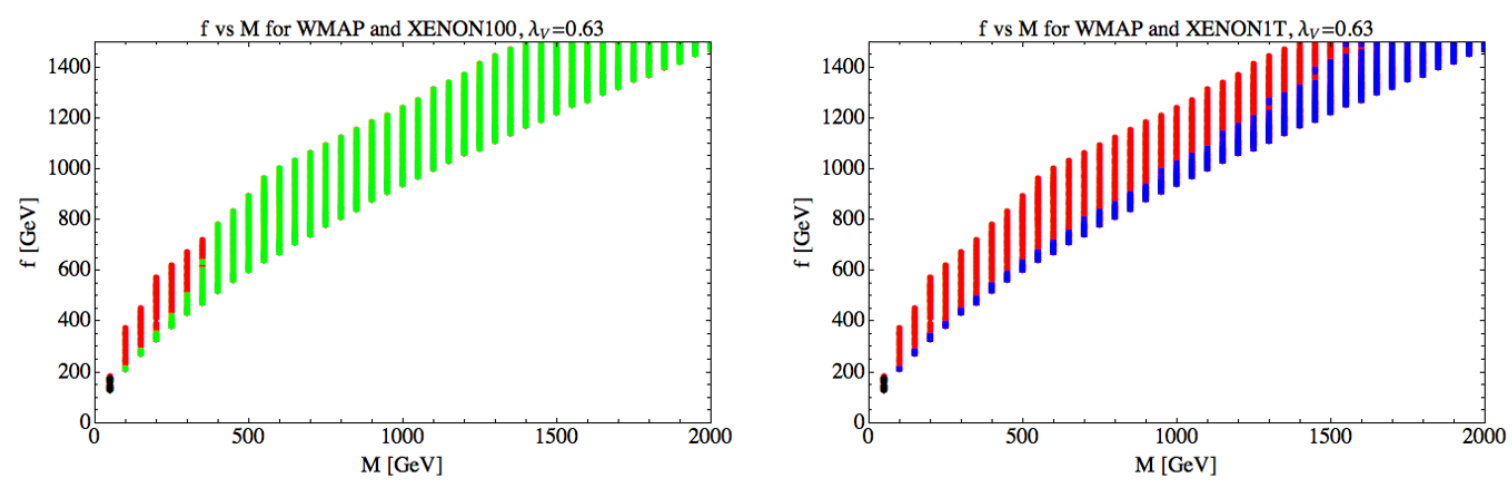

Figure 14. Left: region in the $M-f$ plane allowed by the CDM constraint and allowed (green) or disallowed (red) by the XENON100 data (6.9); black points are disallowed by (3.21). Right: same for the predicted XENON1T exclusion region in red and allowed in blue. We took $q=0, \lambda_{V}=0.63$ and $\left|\lambda_{h}\right|<1$.

The resulting allowed regions in parameter space are given in figure 15 for our benchmark value of $\lambda_{V}=0.63$ as well as for the smaller natural value $\lambda_{V}=0.0023$ derived by NDA (see section 2.2). As can be seen from this figure if $\lambda_{h} \neq 0$ current data excludes DMP masses below $\sim 100 \mathrm{GeV}$, while XENON1T would push this limit above $1 \mathrm{TeV}$. As mentioned earlier small DMP masses are excluded because they lead to DM over-abundance, a consequence of a softening of the cross sections produced by the derivative coupling of the DMP. These limits do not apply when $\lambda_{h} \simeq 0$; in this case low values $(<100 \mathrm{GeV})$ for $M$ and $f$ are allowed; in this case a non-zero value of $q$ can always be found that meets all constraints (see eq. (6.2)). 

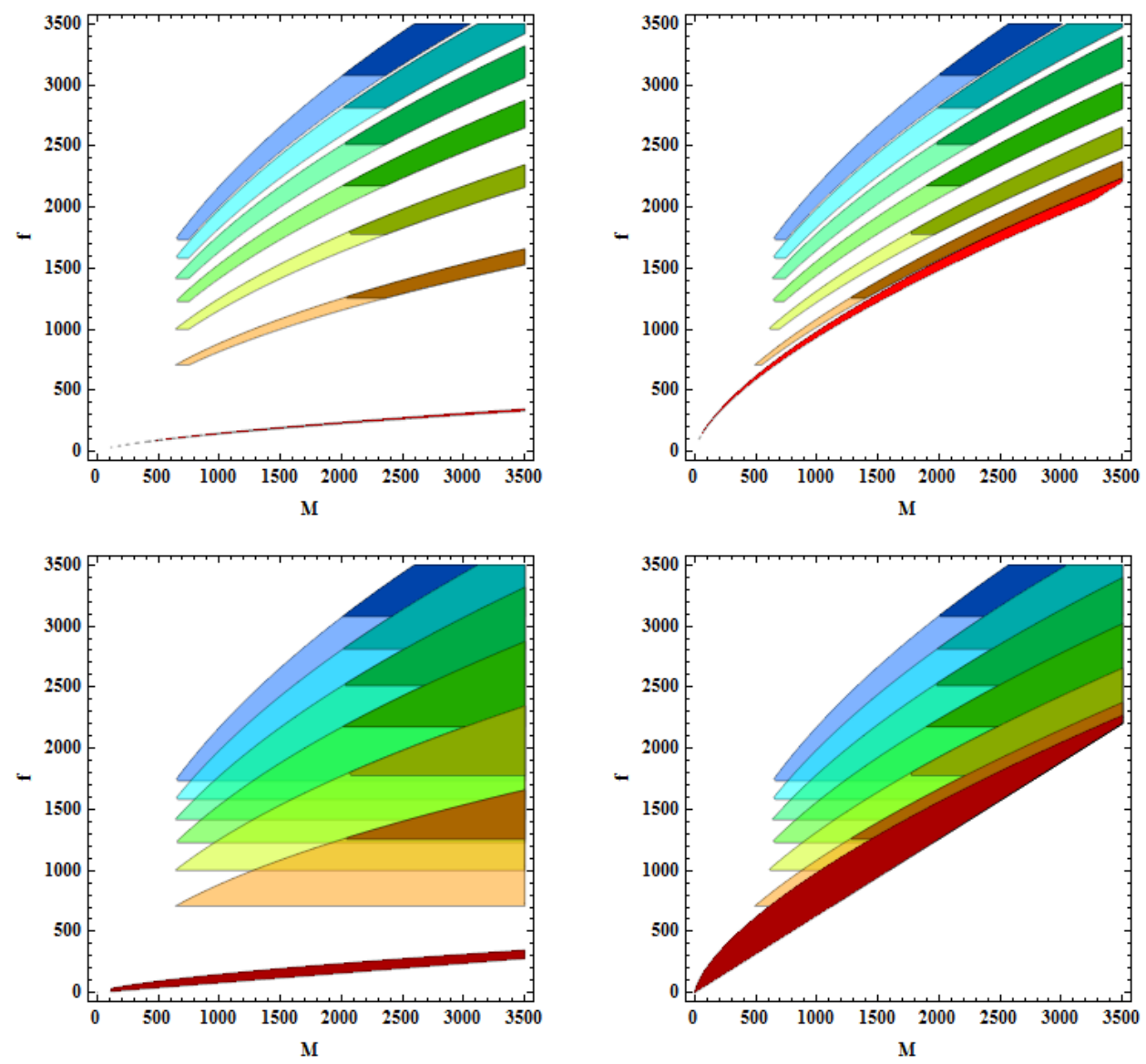

Figure 15. Top left panel: region in the $f-M$ plane allowed by the combined constraints (6.10) when $q=0$ for $\lambda_{V}=0.0023$. The various bands correspond to $\lambda_{h}=\{0,0.5,1,1.5,2,2.5,3\}$ from bottom to top, respectively; the darker regions correspond to those allowed by XENON1T. Top right panel: same for $\lambda_{V}=0.63$. Bottom panels: same as the top panels when $q \neq 0$.

\subsection{Comparison with the standard Higgs-portal results}

Here we present, as mentioned in section 2, a short discussion on the manner in which our results compare with those obtained using the usual Higgs-portal coupling, corresponding to $\lambda_{h}^{\prime} \neq 0$ in (2.14) and $\lambda_{h}=\lambda_{V}=0$. For brevity we will restrict ourselves to the $\mathrm{SU}(2)$ case and take $M>m_{h}$; in this case we can approximate [72]

$$
\langle\sigma v\rangle_{\pi \pi \rightarrow \mathrm{SM}}^{\prime} \simeq \frac{\lambda_{h}^{\prime 2}}{32 \pi M^{2}}+\frac{\lambda_{h}^{\prime 2} v^{2} \Gamma_{h}(2 M)}{32 M^{5}} \simeq\left(\frac{\lambda_{h}^{\prime}}{7.62 M}\right)^{2}, \quad\left(\lambda_{h}=\lambda_{V}=0\right)
$$

where $v=\langle\phi\rangle$ on the right-hand side, $\Gamma_{h}(2 M) \simeq 0.48 \mathrm{TeV}(2 M / 1 \mathrm{TeV})^{3}$ denotes the Higgs particle width when its mass equals $2 M$, and $M$ is in $\mathrm{GeV}$ units in the last expression. The first contribution comes from the $h h$ final state, and the second from the other final states. Note that $\langle\sigma v\rangle_{\mathrm{SM}}^{\prime} \propto M^{-2}$, while $\langle\sigma v\rangle_{\mathrm{SM}} \propto M^{2}$, with the difference a consequence of the derivative coupling of the DMP that leads to the exclusion of the small $M$ region when $\lambda_{h} \neq 0$. 
The freeze-out value of $x, x_{f}=M / T_{f}$ and zero temperature distribution function $Y_{\infty}$ (for each dark pion state) are [65]

$$
x_{f} \simeq \ln \left[c(c+2) a \vartheta_{\mathrm{SM}}^{\prime}\right] ; \quad Y_{\infty} \simeq \frac{x_{f}}{\vartheta_{\mathrm{SM}}^{\prime}}
$$

where

$$
\vartheta_{\mathrm{SM}}^{\prime}=\sqrt{\frac{\pi g(M)}{45 G}} M\langle\sigma v\rangle^{\prime}, \quad\left(\lambda_{h}=\lambda_{V}=0\right)
$$

that are to be compared with (5.18).

It follows form these expressions that for the purposes of calculating the relic abundance, the qualitative difference between the case we have studied here and the standard Higgs-portal results is generated by the $\lambda_{V}$ coupling of the $\Sigma$ - $B_{\mu \nu}$ term in (2.8) (see section 2 for details). The $\lambda_{h}$ coupling generates $s$-wave annihilation into the SM, just as the standard portal coupling does. For large DMP masses (above $\sim 100 \mathrm{GeV}$ ) the fact that this is a derivative coupling is not significant: all cross sections have a minimum CM energy of $2 M$, excluding the zero momentum region where the $\lambda_{h}$ terms differ qualitatively from the standard portal interaction proportional to $\lambda_{h}^{\prime}$.

\section{Conclusions}

We have studied a phenomenological model, where dark matter particles are pseudoGoldstone bosons associated with the spontaneous breaking $\mathcal{G}_{\mathrm{DM}} \rightarrow \mathcal{H}_{\mathrm{DM}}$; we refer to these particles as dark matter "pions", DMP. ${ }^{11}$

The self-couplings and the couplings to the SM for such pionic DM differ from those of conventional scalars due to their chiral nature. We have illustrated the formalism for the case $\mathcal{G}_{\mathrm{DM}}=\mathrm{SU}(2) \times \mathrm{SU}(2), \mathcal{H}_{\mathrm{DM}}=\mathrm{SU}(2)$ for which we have calculated all possible interactions and solved the Boltzmann equations to study the thermal history of such pionic dark matter. We have also derived approximate analytic solutions and shown that they are consistent with the numerical calculations.

Our model of pionic dark matter satisfy relic abundance and direct detection constraint in a large region of parameter space. When the coupling to the Higgs is not too small the DMP mass $M$ is required to lie above $\sim 100 \mathrm{GeV}$, and this lower limit will increase to $\sim 2 \mathrm{TeV}$ if XENON1T does not detect a signal, since the absence of direct detection corresponds to relatively large values of $f^{2} / \lambda_{h}$. For each value of $M$ the DMP decay constant $f$ is moderately constrained to a range of values which is $\sim 200 \mathrm{GeV}$ wide.

Collider signature of such dark matters at LHC is hard to see. The channel to study is essentially jets with missing energy [73], which is similar to many other dark matter model signatures [74]. This requires a careful analysis to see if the existing bound in such channels put further constraints on the DMP parameter space, which lies beyond the scope of this paper. We will consider this in a future publication.

\footnotetext{
${ }^{11}$ As in QCD, there will presumably be baryons in this model (corresponding to solitons in the chiral theory, stabilized by higher derivative terms such as the Skyrme term [42-44]), but though they are SM singlets, they carry DM baryon number, so they do not couple singly to the SM, and they do not look like RH neutrinos.
} 
The DM couples to the SM via $Z, \gamma$ and $h$, therefore it does not distinguish between fermion flavors. In particular there is no mechanism for suppressing the effects of the $\pi$ at XENON experiments and enhancing them at DAMA/LIBRA $[58,59]$.

Though a careful discussion of the indirect detection prospects for this model lies beyond the scope of this publication, it is worth noting that the presence of the $\pi^{3} \gamma$ vertex would imply that a region with sufficiently high concentration of non-relativistic dark pions would act as a source of monochromatic photons of energy $M$ [75]. This is what can occur at the galactic center, in which case the flux corresponding to this process is [76]

$$
\Phi_{\gamma}=\frac{\langle\sigma v\rangle}{4 \pi M^{2}} \int_{\text {l.o.s. }} \rho^{2}(l) d l(\psi),
$$

where $\sigma$ refers to the $\pi \pi \rightarrow \pi \gamma$ cross section (3.7), (3.8), $\rho$ denotes the DM density along the line of sight (l.o.s.), and $\psi$ the angle between the direction of the galactic center and that of observation.

We now assume $M>m_{Z}$ and restrict ourselves to regions in the parameter space where the terms proportional to $\lambda_{V}$ are the dominant contributions to the abundance. In this case we can neglect $\vartheta_{\mathrm{SM}}$ in (5.18); and also $\langle\sigma v\rangle_{\pi \pi \rightarrow \pi \gamma} \simeq c_{\mathrm{w}}^{2}\langle\sigma v\rangle_{\pi \pi \rightarrow \pi V}$, which can be directly related to the relic abundance. We find $\langle\sigma v\rangle \simeq 4.5 \times 10^{-27} \mathrm{~cm}^{3} / \mathrm{s}$ and

$$
\Phi_{\gamma} \simeq 6.54 \times 10^{-11} \mathrm{~s}^{-1} \mathrm{~cm}^{-2}\left(\frac{0.13}{\Omega_{\mathrm{DM}} h^{2}}\right)\left(\frac{M}{100 \mathrm{GeV}}\right)^{-2} J(\psi),
$$

where $J$ is a dimensionless function defined in reference [76] and ranges from about $10^{-2}$ to 100 depending on the density profile and the angle $\psi$. For the FermiLAT detector [77] this gives about $20(M / 100 \mathrm{GeV})^{-2} J$ events per year; it is noteworthy that this result is in rough agreement with the tentative signal obtained from that experiment at an energy of $130 \mathrm{GeV}[78]$.

\section{A Effects on the Boltzmann equations of the SM particle decays to DMP.}

The decay of the SM particles to the DMP require modification of the Boltzmann equation collision term by adding two terms $\mathcal{C}^{h}$ and $\mathcal{C}^{Z}$ corresponding to the $h \rightarrow \pi \pi$ and $Z \rightarrow \pi \pi \pi$ decays. For the first,

$$
\begin{aligned}
\mathcal{C}^{h} & =2 \int d \Pi_{h} d \Pi_{\pi} d \Pi_{\pi}(2 \pi)^{4} \delta^{(4)}\left(p_{h}-p_{\pi}-p_{\pi}\right)\left|\mathcal{A}_{h \rightarrow \pi+\pi}\right|^{2} f_{h}\left(1+f_{\pi}\right)\left(1+f_{\pi}\right) \\
& \simeq N_{h}^{(i)} m_{h} \Gamma(h \rightarrow \pi \pi) \int \frac{d p^{3}}{(2 \pi)^{3} E_{h}} f_{h},
\end{aligned}
$$

where the pre factor of $N_{h}^{(i)}$ corresponds to the number $\pi_{i}$ produced, and we approximated $\left(1+f_{\pi}\right) \simeq 1$. Since $\Gamma$ does not depend on $E_{h}=\sqrt{p^{2}+m_{h}^{2}}$, and using $f_{h}=e^{-E_{h} / T}$ (we assume a vanishing Higgs chemical potential), it follows

$$
\begin{aligned}
\mathcal{C}^{h} & =N_{h}^{(i)} m_{h} \Gamma(h \rightarrow \pi \pi) \int \frac{d p^{3}}{(2 \pi)^{3} E_{h}} e^{-E_{h} / T}=\frac{N_{h}^{(i)}}{2} \frac{m_{h}^{3}}{\pi^{2}} \frac{K_{1}\left(\kappa_{h}\right)}{\kappa_{h}} \Gamma(h \rightarrow \pi \pi) \\
& =N_{h}^{(i)} \Gamma(h \rightarrow \pi \pi) \frac{K_{1}\left(\kappa_{h}\right)}{K_{2}\left(\kappa_{h}\right)} n_{h}^{(\mathrm{eq})}\left(\kappa_{h}\right),
\end{aligned}
$$

with $\kappa_{i}$ defined in (3.4), $\Gamma(h \rightarrow \pi \pi)$ is given in (3.20), and where we used (4.4). 
In complete analogy, the corresponding contribution from $\Gamma \rightarrow \pi \pi \pi$ is

$$
\mathcal{C}^{Z}=\Gamma(Z \rightarrow \pi \pi \pi) \frac{K_{1}\left(\kappa_{Z}\right)}{K_{2}\left(\kappa_{Z}\right)} n_{Z}^{(\mathrm{eq})}\left(\kappa_{Z}\right),
$$

where $\Gamma(Z \rightarrow \pi \pi \pi)$ is given in (3.22). Note that for this decay the final state has a single $\pi_{i}$ (and a $\pi_{ \pm \boldsymbol{\alpha}}$ pair) so the prefactor corresponding to $N_{h}^{(i)}$ is $N_{Z}^{(i)}=1$.

\section{B Kinetics of pure DMP}

Using expressions from section 4 and section 5, and eq. (3.18) the Boltzmann equations for pure DMP scattering are

$$
\begin{aligned}
\frac{d Y_{i}}{d \tau} & =-\sum_{j \neq i}\left(Y_{i}^{2}-Y_{j}^{2}\right)-\sum_{\boldsymbol{\alpha}>0}\left(Y_{i}^{2}-Y_{\boldsymbol{\alpha}} Y_{-\boldsymbol{\alpha}}\right), \\
\frac{d Y_{\boldsymbol{\alpha}}}{d \tau} & =-\sum_{i}\left(Y_{\boldsymbol{\alpha}} Y_{-\boldsymbol{\alpha}}-Y_{i}^{2}\right)-\sum_{\boldsymbol{\beta} \neq \pm \boldsymbol{\alpha}, \boldsymbol{\beta}>0}\left(Y_{\boldsymbol{\alpha}} Y_{-\boldsymbol{\alpha}}-Y_{\boldsymbol{\beta}} Y_{-\boldsymbol{\beta}}\right),
\end{aligned}
$$

where $d \tau=\xi d x$ with

$$
\xi=\sqrt{\frac{\pi g(T)}{45 G}} \frac{M}{x^{2}}\langle\sigma v\rangle_{\pi_{i} \pi_{i} \rightarrow \pi_{\alpha} \pi_{-\alpha}}
$$

and the last factor is explicitly given in (4.8). We solve these equations in two special cases

- Suppose $Y_{i}=Y_{j}=Y_{C}$ for all $i, j$ and $Y_{\boldsymbol{\alpha}}=Y_{\boldsymbol{\beta}}=Y_{R}$ for all $\boldsymbol{\alpha}, \boldsymbol{\beta}$; then

$$
\frac{d Y_{C}}{d \tau}=-\frac{N(N-1)}{2}\left(Y_{C}^{2}-Y_{R}^{2}\right), \quad \frac{d Y_{R}}{d \tau}=-\frac{N-1}{2}\left(Y_{R}^{2}-Y_{C}^{2}\right),
$$

with solutions

$$
Y_{C}=\frac{N^{2} \mathcal{N}}{N^{2}-1}\left(w-\frac{1}{N}\right), \quad Y_{R}=\frac{N^{2} \mathcal{N}}{N^{2}-1}\left(1-\frac{w}{N}\right),
$$

where $\mathcal{N}$ is a constant and

$$
w=\tanh \left(\frac{N(N-1)}{2} \mathcal{N} \tau+\text { const }\right)
$$

In particular, $Y_{C}(\tau=\infty)=Y_{R}(\tau=\infty)=N \mathcal{N} /(N+1)$.

- $N=2$. Using the notation of section 5

$$
\frac{d Y_{o}}{d \tau}=Y_{+} Y_{-}-Y_{o}^{2}, \quad \frac{d Y_{ \pm}}{d \tau}=\frac{1}{2}\left(Y_{o}^{2}-Y_{+} Y_{-}\right)
$$

then $^{12} Y_{o}+Y_{+}+Y_{-}=3 \mathcal{N}=$ const, and $Y_{+}-Y_{-}=6 \mathcal{N} \delta=$ const. Defining now

$$
\eta=\sqrt{\left|1-3 \delta^{2}\right|}, \quad u=\frac{3 \eta \mathcal{N}}{2} \tau+u_{0}, \quad y_{o, \pm}=\frac{Y_{o, \pm}}{\mathcal{N}},
$$

\footnotetext{
${ }^{12}$ Other constants of the motion of the form $\left(c_{+}+c_{-}\right) Y_{o}+2 c_{+} Y_{+}+2 c_{-} Y_{-}$are not independent.
} 
where $u_{0}$ is a constant, the time-dependent solutions for $3 \delta^{2}<1$ are

$$
\begin{array}{ll}
y_{o}=-1+2 \eta \tanh (u) & y_{ \pm}=2 \pm 3 \delta-\eta \tanh (u) \\
& \text { or } \\
y_{o}=-1+2 \eta \operatorname{coth}(u) & y_{ \pm}=2 \pm 3 \delta-\eta \operatorname{coth}(u),
\end{array}
$$

where the second set diverges at $u=0$; in particular, for $\tau \rightarrow \infty$ : $y_{o} \rightarrow-1+2 \eta$, $y_{ \pm} \rightarrow 2 \pm 3 \delta+\eta$ (for $\tau \rightarrow-\infty$ replace $\eta \rightarrow-\eta$ ). For $3 \delta^{2}>1$ the time-dependent solutions become

$$
n_{o}=-1-2 \eta \tan (u), \quad n_{ \pm}=2 \pm 3 \delta+\eta \tan (u),
$$

which diverge for $u=(n+1 / 2) \pi, n \in \mathbb{Z}$. Note that for all the time-dependent solutions there is always an unphysical $\tau$ region where $Y_{o}<0$.

There are also constant solutions

$$
\begin{array}{ll}
y_{o}=-1-2 \eta & y_{ \pm}=2 \pm 3 \delta+\eta \\
y_{o}=-1+2 \eta & y_{ \pm}=2 \pm 3 \delta-\eta
\end{array}
$$

that are real only for $3 \delta^{2} \leq 1$; note that the $\tau$-dependent solutions interpolate between them. Only the second set has a region $(|\delta| \leq 1 / 2)$ where they are all positive, so these correspond to the steady-state solutions.

\section{Acknowledgments}

The work of SB is supported by U.S Department of Energy under Grant No. DE-SC0008541. BM acknowledges the support of the Fulbright Foundation and the Ministry of Science and Technology of the Republic of Croatia under Contract No. 098-0982930-2864.

Open Access. This article is distributed under the terms of the Creative Commons Attribution License (CC-BY 4.0), which permits any use, distribution and reproduction in any medium, provided the original author(s) and source are credited.

\section{References}

[1] J.H. Oort, The force exerted by the stellar system in the direction perpendicular to the galactic plane and some related problems, Bull. Astron. Inst. Netherlands 6 (1932) 249.

[2] F. Zwicky, Die Rotverschiebung von extragalaktischen Nebeln, Helv. Phys. Acta 6 (1933) 110 [inSPIRE].

[3] F. Zwicky, On the Masses of Nebulae and of Clusters of Nebulae, Astrophys. J. 86 (1937) 217 [INSPIRE].

[4] P.J.E. Peebles, Principles of Physical Cosmology, Princeton University Press, (1993).

[5] A. Klypin, J. Holtzman, J. Primack and E. Regos, Structure formation with cold plus hot dark matter, Astrophys. J. 416 (1993) 1 [astro-ph/9305011] [INSPIRE]. 
[6] N. Jarosik et al., Seven-Year Wilkinson Microwave Anisotropy Probe (WMAP) Observations: Sky Maps, Systematic Errors and Basic Results, Astrophys. J. Suppl. 192 (2011) 14 [arXiv: 1001.4744] [INSPIRE].

[7] Planck collaboration, P. Ade et al., Planck 2013 results. XVI. Cosmological parameters, arXiv: 1303.5076 [INSPIRE].

[8] H. Goldberg, Constraint on the Photino Mass from Cosmology, Phys. Rev. Lett. 50 (1983) 1419 [Erratum ibid. 103 (2009) 099905] [INSPIRE].

[9] J.R. Ellis, J. Hagelin, D.V. Nanopoulos, K.A. Olive and M. Srednicki, Supersymmetric Relics from the Big Bang, Nucl. Phys. B 238 (1984) 453 [INSPIRE].

[10] H.-C. Cheng, K.T. Matchev and M. Schmaltz, Radiative corrections to Kaluza-Klein masses, Phys. Rev. D 66 (2002) 036005 [hep-ph/0204342] [INSPIRE].

[11] G. Servant and T.M. Tait, Elastic scattering and direct detection of Kaluza-Klein dark matter, New J. Phys. 4 (2002) 99 [hep-ph/0209262] [INSPIRE].

[12] K. Agashe and G. Servant, Warped unification, proton stability and dark matter, Phys. Rev. Lett. 93 (2004) 231805 [hep-ph/0403143] [INSPIRE].

[13] J. Hubisz and P. Meade, Phenomenology of the littlest Higgs with T-parity, Phys. Rev. D 71 (2005) 035016 [hep-ph/0411264] [INSPIRE].

[14] E. Ma, Variants of the Dark Left-Right Gauge Model: Neutrinos and Scotinos, Phys. Rev. D 79 (2009) 117701 [arXiv:0904.1378] [INSPIRE].

[15] F. Petriello and K.M. Zurek, DAMA and WIMP dark matter, JHEP 09 (2008) 047 [arXiv:0806.3989] [INSPIRE].

[16] K.M. Zurek, Multi-Component Dark Matter, Phys. Rev. D 79 (2009) 115002 [arXiv:0811.4429] [INSPIRE].

[17] D.E. Kaplan, M.A. Luty and K.M. Zurek, Asymmetric Dark Matter, Phys. Rev. D 79 (2009) 115016 [arXiv: 0901.4117] [INSPIRE].

[18] G. Bertone, D. Hooper and J. Silk, Particle dark matter: Evidence, candidates and constraints, Phys. Rept. 405 (2005) 279 [hep-ph/0404175] [INSPIRE].

[19] M. Cvetič, D.A. Demir, J. Espinosa, L. Everett and P. Langacker, Electroweak breaking and the mu problem in supergravity models with an additional U(1), Phys. Rev. D 56 (1997) 2861 [Erratum ibid. D 58 (1998) 119905] [hep-ph/9703317] [INSPIRE].

[20] V. Barger, P. Langacker, I. Lewis, M. McCaskey, G. Shaughnessy and B. Yencho, Recoil Detection of the Lightest Neutralino in MSSM Singlet Extensions, Phys. Rev. D 75 (2007) 115002 [hep-ph/0702036] [INSPIRE].

[21] S. Bhattacharya, J.L. Diaz-Cruz, E. Ma and D. Wegman, Dark Vector-Gauge-Boson Model, Phys. Rev. D 85 (2012) 055008 [arXiv: 1107.2093] [INSPIRE].

[22] J. McDonald, Gauge singlet scalars as cold dark matter, Phys. Rev. D 50 (1994) 3637 [hep-ph/0702143] [INSPIRE].

[23] R. Barbieri, L.J. Hall and V.S. Rychkov, Improved naturalness with a heavy Higgs: An Alternative road to LHC physics, Phys. Rev. D 74 (2006) 015007 [hep-ph/0603188] [INSPIRE].

[24] S. Weinberg, The Quantum Theory of Fields, Cambridge University Press, (2005). 
[25] M.L. Graesser, I.M. Shoemaker and L. Vecchi, Asymmetric WIMP dark matter, JHEP 10 (2011) 110 [arXiv: 1103.2771] [INSPIRE].

[26] R.H. Brandenberger, N. Kaiser, D.N. Schramm and N. Turok, Galaxy and Structure Formation with Hot Dark Matter and Cosmic Strings, Phys. Rev. Lett. 59 (1987) 2371 [INSPIRE].

[27] M. Davis, F. Summers and D. Schlegel, Large scale structure in a universe with mixed hot and cold dark matter, Nature 359 (1992) 393 [INSPIRE].

[28] A. Klypin, J. Holtzman, J. Primack and E. Regos, Structure formation with cold plus hot dark matter, Astrophys. J. 416 (1993) 1 [astro-ph/9305011] [INSPIRE].

[29] T. Hur, D.-W. Jung, P. Ko and J.Y. Lee, Electroweak symmetry breaking and cold dark matter from strongly interacting hidden sector, Phys. Lett. B 696 (2011) 262 [arXiv: 0709.1218] [INSPIRE].

[30] T. Hur and P. Ko, Scale invariant extension of the standard model with strongly interacting hidden sector, Phys. Rev. Lett. 106 (2011) 141802 [arXiv:1103.2571] [INSPIRE].

[31] Y. Bai and R.J. Hill, Weakly Interacting Stable Pions, Phys. Rev. D 82 (2010) 111701 [arXiv: 1005.0008] [INSPIRE].

[32] Y. Bai and P. Schwaller, The Scale of Dark QCD, arXiv:1306.4676 [INSPIRE].

[33] B. Gripaios, A. Pomarol, F. Riva and J. Serra, Beyond the Minimal Composite Higgs Model, JHEP 04 (2009) 070 [arXiv:0902.1483] [INSPIRE].

[34] M. Frigerio, A. Pomarol, F. Riva and A. Urbano, Composite Scalar Dark Matter, JHEP 07 (2012) 015 [arXiv: 1204.2808] [INSPIRE].

[35] S.R. Coleman, J. Wess and B. Zumino, Structure of phenomenological Lagrangians. 1., Phys. Rev. 177 (1969) 2239 [inSPIRE].

[36] C.G. Callan Jr., S.R. Coleman, J. Wess and B. Zumino, Structure of phenomenological Lagrangians. 2., Phys. Rev. 177 (1969) 2247 [INSPIRE].

[37] E. Farhi and L. Susskind, Technicolor, Phys. Rept. 74 (1981) 277 [InSPIRE].

[38] G. 't Hooft, How Instantons Solve the U(1) Problem, Phys. Rept. 142 (1986) 357 [INSPIRE].

[39] J. Gasser and H. Leutwyler, Chiral Perturbation Theory to One Loop, Annals Phys. 158 (1984) 142 [INSPIRE].

[40] J. Gasser and H. Leutwyler, Quark Masses, Phys. Rept. 87 (1982) 77 [INSPIRE].

[41] H. Georgi, Weak Interactions and Modern Particle Theory, Dover Books, (2009).

[42] T. Skyrme, A Nonlinear field theory, Proc. Roy. Soc. Lond. A 260 (1961) 127 [InSPIRE].

[43] G.S. Adkins, C.R. Nappi and E. Witten, Static Properties of Nucleons in the Skyrme Model, Nucl. Phys. B 228 (1983) 552 [inSPIRE].

[44] S. Saito, A Review On The Skyrme Model, in proceedings of Meeting on Few-body Problems In High and Medium Energy Physics, Tsukuba, Japan, 3-5 October 1985, pg. 75-84, INSPIRE.

[45] M. Archidiacono, S. Hannestad, A. Mirizzi, G. Raffelt and Y.Y. Wong, Axion hot dark matter bounds after Planck, JCAP 10 (2013) 020 [arXiv:1307.0615] [INSPIRE]. 
[46] R. Gilmore, Lie Groups, Physics, and Geometry: An Introduction for Physicists, Engineers and Chemists, Cambridge University Press, (2008).

[47] H. Georgi, Lie Algebras In Particle Physics: from Isospin To Unified Theories (Frontiers in Physics), Westview Press, (1999).

[48] J. March-Russell, S.M. West, D. Cumberbatch and D. Hooper, Heavy Dark Matter Through the Higgs Portal, JHEP 07 (2008) 058 [arXiv: 0801.3440] [INSPIRE].

[49] A. Drozd, B. Grzadkowski and J. Wudka, Multi-Scalar-Singlet Extension of the Standard Model - the Case for Dark Matter and an Invisible Higgs Boson, JHEP 04 (2012) 006 [arXiv: 1112.2582] [INSPIRE].

[50] J. Wess and B. Zumino, Consequences of anomalous Ward identities, Phys. Lett. B 37 (1971) 95 [INSPIRE].

[51] E. Witten, Global Aspects of Current Algebra, Nucl. Phys. B 223 (1983) 422 [InSPIRE].

[52] R. Jackiw, Field Theoretic Investigations In Current Algebra, in Current Algebra and Anomalies, S. Treiman ed., (1986) pg. 81-210, inSPIRE.

[53] A. Manohar and H. Georgi, Chiral Quarks and the Nonrelativistic Quark Model, Nucl. Phys. B 234 (1984) 189 [INSPIRE].

[54] R.S. Chivukula, M.J. Dugan and M. Golden, Electroweak corrections in technicolor reconsidered, Phys. Lett. B 292 (1992) 435 [hep-ph/9207249] [INSPIRE].

[55] H. Georgi, Generalized dimensional analysis, Phys. Lett. B 298 (1993) 187 [hep-ph/9207278] [INSPIRE].

[56] ATLAS collaboration, Observation of a new particle in the search for the Standard Model Higgs boson with the ATLAS detector at the LHC, Phys. Lett. B 716 (2012) 1 [arXiv: 1207.7214] [INSPIRE].

[57] CMS collaboration, Observation of a new boson at a mass of $125 \mathrm{GeV}$ with the CMS experiment at the LHC, Phys. Lett. B 716 (2012) 30 [arXiv:1207.7235] [INSPIRE].

[58] R. Bernabei et al., DAMA/LIBRA results and perspectives, arXiv:1301.6243 [INSPIRE].

[59] DAMA, LIBRA collaborations, R. Bernabei et al., New results from DAMA/LIBRA, Eur. Phys. J. C 67 (2010) 39 [arXiv: 1002.1028] [InSPIRE].

[60] CDMS collaboration, Z. Ahmed et al., Search for Weakly Interacting Massive Particles with the First Five-Tower Data from the Cryogenic Dark Matter Search at the Soudan Underground Laboratory, Phys. Rev. Lett. 102 (2009) 011301 [arXiv:0802.3530] [INSPIRE].

[61] CDMS-II collaboration, Z. Ahmed et al., Dark Matter Search Results from the CDMS II Experiment, Science 327 (2010) 1619 [arXiv:0912.3592] [INSPIRE].

[62] LUX collaboration, D. Akerib et al., First results from the LUX dark matter experiment at the Sanford Underground Research Facility, arXiv:1310.8214 [INSPIRE].

[63] XENON100 collaboration, E. Aprile et al., Implications on Inelastic Dark Matter from 100 Live Days of XENON100 Data, Phys. Rev. D 84 (2011) 061101 [arXiv:1104.3121] [INSPIRE].

[64] XENON100 collaboration, E. Aprile et al., Dark Matter Results from 100 Live Days of XENON100 Data, Phys. Rev. Lett. 107 (2011) 131302 [arXiv:1104.2549] [INSPIRE].

[65] E. W. Kolb and M. S. Turner, The Early universe, Westview Press, (1994). 
[66] S. Dodelson, Modern cosmology, Academic Press, Amsterdam, Netherlands (2003).

[67] S. Weinberg, Gravitation and Cosmology: Principles and Applications of the General Theory of Relativity, John Wiley \& Sons Inc., (1972).

[68] M. Frigerio, T. Hambye and E. Masso, Sub-GeV dark matter as pseudo-Goldstone from the seesaw scale, Phys. Rev. X 1 (2011) 021026 [arXiv:1107.4564] [INSPIRE].

[69] M. Laine and Y. Schröder, Quark mass thresholds in QCD thermodynamics, Phys. Rev. D 73 (2006) 085009 [hep-ph/0603048] [INSPIRE].

[70] Particle Data Group collaboration, J. Beringer et al., Review of Particle Physics (RPP), Phys. Rev. D 86 (2012) 010001 [inSPIRE].

[71] G. Bélanger, F. Boudjema, A. Pukhov and A. Semenov, Dark matter direct detection rate in a generic model with MicrOMEGAs 2.2, Comput. Phys. Commun. 180 (2009) 747 [arXiv:0803.2360] [INSPIRE].

[72] B. Grzadkowski and J. Wudka, Pragmatic approach to the little hierarchy problem: the case for Dark Matter and neutrino physics, Phys. Rev. Lett. 103 (2009) 091802 [arXiv:0902.0628] [INSPIRE].

[73] P.J. Fox, R. Harnik, J. Kopp and Y. Tsai, Missing Energy Signatures of Dark Matter at the LHC, Phys. Rev. D 85 (2012) 056011 [arXiv:1109.4398] [InSPIRE].

[74] A. Djouadi, O. Lebedev, Y. Mambrini and J. Quevillon, Implications of LHC searches for Higgs-portal dark matter, Phys. Lett. B 709 (2012) 65 [arXiv:1112.3299] [InSPIRE].

[75] L. Bergstrom and H. Snellman, Observable Monochromatic Photons From Cosmic Photino Annihilation, Phys. Rev. D 37 (1988) 3737 [INSPIRE].

[76] L. Bergstrom, P. Ullio and J.H. Buckley, Observability of gamma-rays from dark matter neutralino annihilations in the Milky Way halo, Astropart. Phys. 9 (1998) 137 [astro-ph/9712318] [INSPIRE].

[77] LAT collaboration, W. Atwood et al., The Large Area Telescope on the Fermi Gamma-ray Space Telescope Mission, Astrophys. J. 697 (2009) 1071 [arXiv:0902.1089] [INSPIRE].

[78] T. Bringmann, X. Huang, A. Ibarra, S. Vogl and C. Weniger, Fermi LAT Search for Internal Bremsstrahlung Signatures from Dark Matter Annihilation, JCAP 07 (2012) 054 [arXiv: 1203.1312] [INSPIRE]. 